\title{
Total positivity from the exponential Riordan arrays *
}

\author{
Bao-Xuan Zhu \\ School of Mathematics and Statistics, Jiangsu Normal University, Xuzhou 221116, PR China
}

\begin{abstract}
Log-concavity and almost log-convexity of the cycle index polynomials were proved by Bender and Canfield [J. Combin. Theory Ser. A 74 (1996)]. Schirmacher [J. Combin. Theory Ser. A 85 (1999)] extended them to $q$-log-concavity and almost $q$-log-convexity. Motivated by these, we consider the stronger properties total positivity from the Toeplitz matrix and Hankel matrix.

By using exponential Riordan array methods, we give some criteria for total positivity of the triangular matrix of coefficients of the generalized cycle index polynomials, the Toeplitz matrix and Hankel matrix of the polynomial sequence in terms of the exponential formula, the logarithmic formula and the fractional formula, respectively.

Finally, we apply our criteria to some triangular arrays satisfying some recurrence relations, including Bessel triangles of two kinds and their generalizations, the Lah triangle and its generalization, the idempotent triangle and some triangles related to binomial coefficients, rook polynomials and Laguerre polynomials. We not only get total positivity of these lower-triangles, and $q$-Stieltjes moment properties and 3- $q$-log-convexity of their row-generating functions, but also prove that their triangular convolutions preserve Stieltjes moment property. In particular, we solve a conjecture of Sokal on $q$-Stieltjes moment property of rook polynomials.
\end{abstract}

MSC: 05A20; 05A15; 11B83; 15B36; 44A60

Keywords: Exponential generating functions; Riordan arrays; Recurrence relations; Total positivity; Hankel matrices; Toeplitz matrices; Stieltjes moment property; Convolutions; Continued fractions; 3- $q$-log-convexity; Bessel numbers; Lah numbers; Idempotent numbers; Rook polynomials; Laguerre polynomials

\section{Contents}

1 Introduction $\quad 2$

1.1 Cycle index polynomials and Sheffer polynomials . . . . . . . . . . . 2

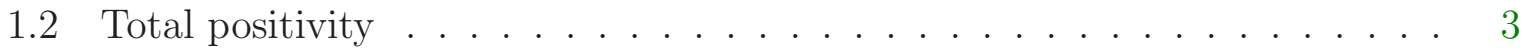

1.3 Extension of the cycle index polynomials . . . . . . . . . . . . . 5

*Supported partially by the National Natural Science Foundation of China (Nos. 11971206, 12022105) and the Natural Science Foundation for Distinguished Young Scholars of Jiangsu Province (No. BK20200048).

Email address: bxzhu@jsnu.edu.cn (B.-X. Zhu) 
2 Total positivity of the Toeplitz matrix 4

3 Exponential Riordan arrays and total positivity 9

3.1 Definition and properties of exponential Riordan arrays . . . . . . . . . . . 9

3.2 Total positivity of exponential Riordan arrays . . . . . . . . . . . . . . . 11

3.3 Total positivity of the Hankel matrix of the 0 th column . . . . . . . . . . . 15

4 Applications 20

4.1 A generalization of Bessel numbers of the second kind . . . . . . . . . . . . 21

4.2 A generalization of Bessel numbers of the first kind . . . . . . . . . . . . . 22

4.3 A generalization of Lah numbers . . . . . . . . . . . . . . . . . . . 27

4.4 Rook polynomials and signless Laguerre polynomials . . . . . . . . . . . 32

4.5 Idempotent numbers . . . . . . . . . . . . . . . . . . . . 33

4.6 Some numbers related to binomial coefficients . . . . . . . . . . . . 34

5 Acknowledgements $\quad 35$

\section{Introduction}

\subsection{Cycle index polynomials and Sheffer polynomials}

Let $x_{1}, x_{2}, \ldots$ be a sequence of nonnegative real numbers and define $A_{n}\left(x_{1}, x_{2}, \ldots, x_{n}\right)$ and $P_{n}\left(x_{1}, x_{2}, \ldots, x_{n}\right)$ by

$$
\sum_{n \geq 0} A_{n}\left(x_{1}, x_{2}, \ldots, x_{n}\right) t^{n}=\sum_{n \geq 0} P_{n}\left(x_{1}, x_{2}, \ldots, x_{n}\right) \frac{t^{n}}{n !}=\exp \left(\sum_{j \geq 1} x_{j} \frac{t^{j}}{j}\right) .
$$

Then each $A_{n}\left(x_{1}, x_{2}, \ldots, x_{n}\right)$ is a polynomial in the variables $x_{j}$ with $1 \leq j \leq n$. This has a well-known combinatorial significance [20]: Let $\Sigma_{n}$ denote the symmetric group and let $N_{j}(\sigma)$ be the number of $j$-cycles in the permutation $\sigma$. Then

$$
A_{n}\left(x_{1}, x_{2}, \ldots, x_{n}\right)=\frac{P_{n}\left(x_{1}, x_{2}, \ldots, x_{n}\right)}{n !}=\frac{1}{n !} \sum_{\sigma \in \Sigma_{n}} x_{1}^{N_{1}(\sigma)} x_{2}^{N_{2}(\sigma)} \cdots x_{n}^{N_{n}(\sigma)} .
$$

This $A_{n}\left(x_{1}, x_{2}, \ldots, x_{n}\right)$ is called the cycle index polynomial generally due to Pólya [42], although in fact appearing in earlier work of Redfield [44]. Bender and Canfield [5] proved that if the positive sequence $\left(x_{n}\right)_{n \geq 0}$ with $x_{0}=1$ is log-concave then $\left(A_{n}\left(x_{1}, x_{2}, \ldots, x_{n}\right)\right)_{n \geq 0}$ is log-concave and $\left(P_{n}\left(x_{1}, x_{2}, \ldots, x_{n}\right)\right)_{n \geq 0}$ is log-convex. In addition, it was extended to the much stronger properties $q$-log-concavity and $q$-log-convexity, i.e., for $n \geq m$,

$$
\begin{aligned}
A_{n}\left(x_{1}, \ldots, x_{n}\right) A_{m}\left(x_{1}, \ldots, x_{m}\right)-A_{n+1}\left(x_{1}, \ldots, x_{n+1}\right) A_{m-1}\left(x_{1}, \ldots, x_{m-1}\right) & \in \mathbb{N}[\mathcal{X}], \\
P_{n+1}\left(x_{1}, \ldots, x_{n+1}\right) P_{m-1}\left(x_{1}, \ldots, x_{m-1}\right)-P_{n}\left(x_{1}, \ldots, x_{n}\right) P_{m}\left(x_{1}, \ldots, x_{m}\right) & \in \mathbb{N}[\mathcal{X}],
\end{aligned}
$$

where $\mathcal{X}=\left\{x_{1}, x_{2}, \ldots\right\} \bigcup\left\{x_{j} x_{k}-x_{j-1} x_{k+1}: 1 \leq j \leq k\right\}$ and $\mathbb{N}[\mathcal{X}]$ denotes the ring of polynomials in the indeterminates $\mathcal{X}$ with nonnegative integer coefficients, see Bender and Canfield [5] and Schirmacher [52]. 
The model in (1.1) also has a probabilistic interpretation from compound Poisson, see [34]. Its log-concavity and log-convexity are very significant in probability and statistics since they play a crucial role in the class of infinitely divisible distributions, one of the most important probability distributions in both theory and applications, see [25, 26, 51].

In addition, the sequences and functions in (1.1) are closely related to Sheffer polynomials $[1,3,53]$. A polynomial $S_{n}(q)=\sum_{k=0}^{n} S_{n, k} q^{k}$ is called the Sheffer polynomial if

$$
\sum_{n \geq 0} S_{n}(q) \frac{t^{n}}{n !}=g(t) \exp (f(t) q),
$$

where $g(0) f^{\prime}(0) \neq 0$ and $f(0)=0$ (cf. [47, 48]). It was proved that many polynomials including Bernoulli polynomials, Euler polynomials, Hermite polynomials, Laguerre polynomials, Poisson-Charlier polynomials and Meixner polynomials are all Sheffer polynomials. Sheffer polynomials can be studied from umbral calculus, which has been used in different areas of mathematics, like approximation theory, analysis, combinatorics, statistics, and so on. For more details on the theory of umbral calculus and Sheffer polynomials, we refer the reader to $[47,49]$.

Motivated by the $q$-log-concavity of $\left(A_{n}\left(x_{1}, x_{2}, \ldots, x_{n}\right)\right)_{n \geq 0}$ and $q$-log-convexity of $\left(P_{n}\left(x_{1}, x_{2}, \ldots, x_{n}\right)\right)_{n \geq 0}$ in the first paragraph, the aim of this paper is to extend them to total positivity of matrices in terms of the Sheffer model in (1.3). Let us recall total positivity of matrices in the following.

\subsection{Total positivity}

Total positivity of matrices is an important and powerful concept that arises often in various branches of mathematics, see the monographs [30, 41] for more details.

Let $M=\left[m_{n, k}\right]_{n, k \geq 0}$ be a matrix of real numbers. It is totally positive (TP for short) if all its minors are nonnegative. It is totally positive of order $r$ ( $T P_{r}$ for short) if all minors of order $k \leq r$ are nonnegative. For a sequence $\alpha=\left(a_{k}\right)_{k>0}$, denote its Toeplitz matrix by $\Gamma(\alpha)=\left[a_{i-j}\right]_{i, j \geq 0}$ and Hankel matrix by $H(\alpha)=\left[a_{i+j}\right]_{i, j \geq 0}$, which play an important role in different fields.

The sequence $\alpha$ is called a Pólya frequency (PF, for short) sequence if its infinite Toeplitz matrix $\Gamma(\alpha)$ is TP. PF sequences have many nice properties (Karlin [30]). In classical analysis, PF sequences are closely related to real rootedness of polynomials and entire functions. For example, the fundamental representation theorem for PF sequences states that a sequence $a_{0}=1, a_{1}, a_{2}, \ldots$ of real numbers is PF if and only if its generating function has the form

$$
\sum_{n \geq 0} a_{n} z^{n}=\frac{\prod_{j \geq 1}\left(1+\alpha_{j} z\right)}{\prod_{j \geq 1}\left(1-\beta_{j} z\right)} e^{\gamma z}
$$

in some open disk centered at the origin, where $\alpha_{j}, \beta_{j}, \gamma \geq 0$ and $\sum_{j \geq 1}\left(\alpha_{j}+\beta_{j}\right)<+\infty$, see Karlin [30, p. 412] for instance. In particular, a finite sequence of nonnegative numbers is $\mathrm{PF}$ if and only if its generating function has only real zeros ([30, p. 399]). We also call the function $\sum_{n>0} a_{n} z^{n}$ a $\mathrm{PF}\left(\right.$ resp. $\left.\mathrm{PF}_{r}\right)$ function if $\Gamma(\alpha)$ is $\mathrm{TP}\left(\operatorname{resp} . \mathrm{TP}_{r}\right)$. The sequence $\alpha$ is called log-concave if $a_{k-1} a_{k+1} \leq a_{k}^{2}$ for all $k \geq 1$. Clearly, a sequence of positive numbers is log-concave if and only if $\Gamma(\alpha)$ is $\mathrm{TP}_{2}$. Thus it is natural to consider a question whether results for log-concavity can be extended to Pólya frequency property. 
We refer the reader to Brändén [6, 7], Brenti [8, 9, 10], Wang-Yeh [62] and Zhu [67] on the PF property in combinatorics.

The sequence $\alpha$ is a Stieltjes moment (SM for short) sequence if it has the form

$$
a_{k}=\int_{0}^{+\infty} x^{k} d \mu(x),
$$

where $\mu$ is a non-negative measure on $[0,+\infty)$ (see $[41$, Theorem 4.4] for instance). It is well known that $\alpha$ is a Stieltjes moment sequence if and only if its Hankel matrix $H(\alpha)$ is TP [41]. The Stieltjes moment problem is one of classical moment problems and arises naturally in many branches of mathematics [54, 64]. The sequence $\alpha$ is called log-convex if $a_{k-1} a_{k+1} \geq a_{k}^{2}$ for all $k \geq 1$. Clearly, a sequence of positive numbers is log-convex if and only if $H(\alpha)$ is $\mathrm{TP}_{2}$. In consequence, $\mathrm{SM}$ property is much stronger than log-convexity. In addition, log-convexity of many combinatorial sequences can be extended to SM property. We refer the reader to Liu and Wang [28] and Zhu [66] for log-convexity and Wang and Zhu [63] and Zhu[70, 71] for SM property.

For brevity, we use the following notation

(i) $\mathbb{R}$ : the set of all real numbers; $\quad \mathbb{R}^{\geq 0}$ : the set of all nonnegative real numbers;

(ii) $\mathbb{N}^{+}$: the set of all positive integer numbers, and $\mathbb{N}=\mathbb{N}^{+} \cup\{0\}$.

If $\mathbf{x}=\left(x_{i}\right)_{i \in I}$ is a set of indeterminates, we denote by $\mathbb{R}[\mathbf{x}]$ (resp. $\mathbb{R}[[\mathbf{x}]]$ ) the ring of polynomials (resp. formal power series) in the indeterminates $\mathbf{x}$ with coefficients in $\mathbb{R}$. A matrix $M$ with entries in $\mathbb{R}[\mathbf{x}]$ is called $\boldsymbol{x}$-totally positive ( $\boldsymbol{x}$-TP for short) if all its minors are polynomials with nonnegative coefficients in the indeterminates $\mathbf{x}$ and is called $\boldsymbol{x}$-totally positive of order $r\left(\boldsymbol{x}\right.$ - $T P_{r}$ for short) if all its minors of order $k \leq r$ are polynomials with nonnegative coefficients in the indeterminates $\mathbf{x}$. A polynomial sequence $\left(\alpha_{n}(\mathbf{x})\right)_{n \geq 0}$ in $\mathbb{R}[\mathbf{x}]$ is called an $\boldsymbol{x}$-Stieltjes moment (x-SM for short) sequence (we also call it coefficientwise Hankel-total positive, see [40]) if its associated infinite Hankel matrix is $\mathbf{x}$-totally positive. It is called $\boldsymbol{x}$-log-convex ( $\mathbf{x}$-LCX for short) if

$$
\alpha_{n+1}(\mathbf{x}) \alpha_{n-1}(\mathbf{x})-\alpha_{n}(\mathbf{x})^{2} \in \mathbb{R}^{\geq 0}[\mathbf{x}]
$$

for all $n \in \mathbb{N}^{+}$and is called strongly $\boldsymbol{x}$-log-convex (x-SLCX for short) if

$$
\alpha_{n+1}(\mathbf{x}) \alpha_{m-1}(\mathbf{x})-\alpha_{n}(\mathbf{x}) \alpha_{m}(\mathbf{x}) \in \mathbb{R}^{\geq 0}[\mathbf{x}]
$$

for all $n \geq m \geq 1$. Clearly, an $\mathbf{x}$-SM sequence is both $\mathbf{x}$-SLCX and $\mathbf{x}$-LCX. Define an operator $\mathcal{L}$ by

$$
\mathcal{L}\left[\alpha_{i}(\mathbf{x})\right]:=\alpha_{i-1}(\mathbf{x}) \alpha_{i+1}(\mathbf{x})-\alpha_{i}(\mathbf{x})^{2}
$$

for $i \in \mathbb{N}^{+}$. Then the $\mathbf{x}$-log-convexity of $\left(\alpha_{i}(\mathbf{x})\right)_{i \geq 0}$ is equivalent to $\mathcal{L}\left[\alpha_{i}(\mathbf{x})\right] \in \mathbb{R}^{\geq 0}[\mathbf{x}]$ for all $i \in \mathbb{N}^{+}$. In general, we say that $\left(\alpha_{i}(\mathbf{x})\right)_{i \geq 0}$ is $k$-x-log-convex if $\mathcal{L}^{m}\left[\alpha_{i}(\mathbf{x})\right] \in \mathbb{R}^{\geq 0}[\mathbf{x}]$ for all $m \leq k$, where $\mathcal{L}^{m}=\mathcal{L}\left(\mathcal{L}^{m-1}\right)$. The sequence $\left(\alpha_{i}(\mathbf{x})\right)_{i \geq 0}$ is called infinitely $\boldsymbol{x}$-log-convex if it is $k$-x-log-convex for every $k \in \mathbb{N}$. See $[12,31,33]$ for $\mathbf{x}$-log-concavity, $[10,50]$ for $\mathbf{x}-\mathrm{PF},[16,28,66,67,68]$ for $\mathbf{x}$-LCX or $\mathbf{x}-S L C X$, and $[39,40,63,70,72,73]$ for $\mathbf{x}$-SM.

In $[40,73]$, the authors proved many results for coefficientwise total positivity and coefficientwise Hankel-total positivity. In a certain sense, this paper can be viewed as to keep the study in $[40,73]$. We will present our results in the following. 


\subsection{Extension of the cycle index polynomials}

As an extension of log-concavity of the cycle index polynomials, we present a result for total positivity as follows.

Theorem 1.1. Let $g(t) \in \mathbb{R}[[t]]$ and $\boldsymbol{x}=\left(x_{i}\right)_{i \geq 1} \subseteq \mathbb{R}$. Define a generalized cycle index polynomial sequence $\left(\mathcal{A}_{n}(\boldsymbol{x})\right)_{n \geq 0}$ by

$$
\sum_{n \geq 0} \mathcal{A}_{n}(\boldsymbol{x}) t^{n}=g(t) \exp \left(\sum_{j \geq 1} x_{j} \frac{t^{j}}{j}\right) .
$$

Assume that $g(t)$ is a $P F_{r}$ function. If there exists a nonnegative sequence $\left(\lambda_{i}\right)_{i \geq 1}$ such that $x_{n}=\sum_{i \geq 1} \lambda_{i}^{n}$ for $n \geq 1$, then the Toeplitz matrix $\left[\mathcal{A}_{i-j}(\boldsymbol{x})\right]_{i, j \geq 0}$ is $\operatorname{TP}_{r}$.

Let functions $f(t) \in \mathbb{R}[[t]]$ and $g(t) \in \mathbb{R}[[t]]$, where $f(0)=0$ and $f^{\prime}(0) g(0) \neq 0$. Define a cycle-index-triangle $\left[A_{n, k}(f, g)\right]_{n, k \geq 0}$ and a Sheffer-triangle $\left[S_{n, k}(f, g)\right]_{n, k \geq 0}$ by

$$
g(t) \exp (q f(t)):=1+\sum_{n \geq 1}\left(\sum_{k=1}^{n} A_{n, k}(f, g) q^{k}\right) t^{n}=1+\sum_{n \geq 1}\left(\sum_{k=1}^{n} S_{n, k}(f, g) q^{k}\right) \frac{t^{n}}{n !} .
$$
by

Similarly, define a logarithmic triangle $\left[L_{n, k}(f)\right]_{n, k \geq 0}$ and a fractional triangle $\left[\widetilde{L}_{n, k}(f)\right]_{n, k \geq 0}$

$$
\begin{aligned}
\log (1-q f(t)): & =\sum_{n \geq 1}-\left(\sum_{k=1}^{n} L_{n, k}(f) q^{k}\right) \frac{t^{n}}{n !} \\
\frac{1}{1-q f(t)}: & =1+\sum_{n \geq 1}\left(\sum_{k=1}^{n} \widetilde{L}_{n, k}(f) q^{k}\right) \frac{t^{n}}{n !}
\end{aligned}
$$

More and more combinatorial triangles are proved to be totally positive, for example, the triangle $\left[A_{n, k}\right]$ in $[10]$, which satisfies the recurrence

$$
A_{n, k}=x_{n} A_{n-1, k}+y_{n} A_{n-1-t, k-1}+z_{n} A_{n-t, k-1},
$$

the Pascal triangle [30, p.137], recursive matrices [17], Riordan arrays [18, 19], the JacobiStirling triangle [35], Delannoy-like triangles [37], Catalan-Stieltjes matrices [38], Narayana triangles of types $A$ and $B$ [61], and the generalized Jacobi-Stirling triangle [67]. We refer the reader to $[8,23,57]$ for more total positivity results in combinatorics. Let $\bar{f}(t)$ denote the compositional inverse of the function $f(t)$ (that is $f(\bar{f}(t))=\bar{f}(f(t))=t$ ) and let $\bar{f}^{\prime}(t)$ denote the derivative function of $\bar{f}(t)$. We get the following result for total positivity of triangular matrices.

Theorem 1.2. Let $\left\{r_{1}, r_{2}\right\} \subseteq \mathbb{N}^{+}$and $g(t)$ be a $P F_{r_{1}}$ function. If one of $f(t)$ and $1 / \bar{f}^{\prime}(t)$ is a $P F_{r_{2}}$ function and let $r=\min \left\{r_{1}, r_{2}\right\}$, then

(i) both $\left[S_{n, k}(f, g)\right]_{n, k \geq 0}$ and $\left[S_{n, k}(f, g(f))\right]_{n, k \geq 0}$ are $T P_{r}$;

(ii) both $\left[A_{n, k}(f, g)\right]_{n, k \geq 0}$ and $\left[A_{n, k}(f, g(f))\right]_{n, k \geq 0}$ are $T P_{r}$; 
(iii) $\left[L_{n, k}(f)\right]_{n, k \geq 0}$ is $T P_{r}$;

(iv) $\left[\widetilde{L}_{n, k}(f)\right]_{n, k \geq 0}$ is $T P_{r}$.

Further, let functions $f(t)=\sum_{i \geq 1} f_{i} \frac{t^{i}}{i !} \in \mathbb{R}[[t]]$ and $g(t)=\sum_{i \geq 0} g_{i} \frac{t^{i}}{i !} \in \mathbb{R}[[t]]$, where $f_{1} \neq 0$ and $g_{0} \neq 0$. For $n \geq 1$, denote the Sheffer polynomial by

$$
S_{n}(f, g ; q)=\sum_{k=1}^{n} S_{n, k}(f, g) q^{k}
$$

and $S_{0}(f, g ; q)=1$. Similarly, define a logarithmic polynomial $L_{n}(f ; q)$ and a fractional polynomial $\widetilde{L}_{n}(f ; q)$ by

$$
L_{n}(f ; q):=\sum_{k=1}^{n} L_{n, k}(f) q^{k}, \quad \widetilde{L}_{n}(f ; q):=\sum_{k=1}^{n} \widetilde{L}_{n, k}(f) q^{k}
$$

for $n \geq 1$, where $L_{0}(f ; q)=1$ and $\widetilde{L}_{0}(f ; q)=1$. For a polynomial $P_{n}(q)$ of degree $n$, we denote by $P_{n}^{*}(q)=q^{n} P_{n}(1 / q)$ its reciprocal polynomial.

For Hankel-total-positivity, we get the result as follows.

Theorem 1.3. Let $g(t)=\exp (\lambda f(t))$. If $1 / \bar{f}^{\prime}(t)$ is a PF function, then

(i) the Hankel matrices $\left[S_{i+j}(f, g ; q)\right]_{i, j \geq 0}$ and $\left[S_{i+j}^{*}(f, g ; q)\right]_{i, j \geq 0}$ is $(\lambda, q)-T P_{r}$;

(ii) the Hankel matrix $\left[f_{i+j+1}\right]_{i, j \geq 0}$ is $T P_{r}$.

Let $A=\left[a_{n, k}\right]_{n, k \geq 0}$ be an infinite matrix. For $n \in \mathbb{N}$, define the $A$-convolution

$$
z_{n}=\sum_{k=0}^{n} a_{n k} x_{k} y_{n-k} .
$$

We say that (1.10) preserves the SM property: if both $\left(x_{n}\right)_{n \geq 0}$ and $\left(y_{n}\right)_{n \geq 0}$ are Stieltjes moment sequences, then so is $\left(z_{n}\right)_{n \geq 0}$.

Using positive definiteness of the quadratic form in linear algebra, Pólya and Szegö [43, Part VII, Theorem 42] proved for $n \in \mathbb{N}$ that the binomial convolution

$$
z_{n}=\sum_{k=0}^{n}\left(\begin{array}{l}
n \\
k
\end{array}\right) x_{k} y_{n-k}
$$

preserves the SM property in $\mathbb{R}$. Recently, Wang and the author [63] got more triangular convolutions including the Stirling convolution of the second kind, the Eulerian convolution and so on, preserving the SM property. In addition, the next sufficient condition for the triangular convolution preserving the SM property was provided in [63].

Lemma 1.4. [63] For $n \in \mathbb{N}$, let $A_{n}(q)=\sum_{k=0}^{n} a_{n, k} q^{k}$ be the $n$th row generating function of the matrix $A=\left[a_{n, k}\right]_{n, k}$. Assume that $\left(A_{n}(q)\right)_{n \geq 0}$ is a Stieltjes moment sequence for any fixed $q \geq 0$. Then the A-convolution (1.10) preserves the SM property. 
Clearly, if the sequence $\left(A_{n}(q)\right)_{n \geq 0}$ is $q$-SM, then $\left(A_{n}(q)\right)_{n \geq 0}$ is a Stieltjes moment sequence for any fixed $q \geq 0$. In addition, it also implies 3- $q$-log-convexity of $\left(A_{n}(q)\right)_{n \geq 0}$ in terms of the next result.

Lemma 1.5. $[69,73]$ For a polynomial sequence $\left(A_{n}(\boldsymbol{x})\right)_{n \geq 0}$, if the Hankel matrix $\left[A_{i+j}(\boldsymbol{x})\right]_{i, j \geq 0}$ is $\boldsymbol{x}$ - $T P_{4}$, then $\left(A_{n}(\boldsymbol{x})\right)_{n \geq 0}$ is 3-x-log-convex.

Then we have the following results.

Theorem 1.6. Let $g(t)=\exp (\lambda f(t))$. If $1 / \bar{f}^{\prime}(t)$ is a PF function, then we have

(i) both $\left(S_{n}(f, g ; q)\right)_{n \geq 0}$ and $\left(S_{n}^{*}(f, g ; q)\right)_{n \geq 0}$ are $(\lambda, q)$-SM and 3 - $(\lambda, q)$-log-convex;

(ii) $\left(f_{n+1}\right)_{n \geq 0}$ is $S M$ and 3-log-convex;

(iii) $\left[S_{n, k}(f, g)\right]_{n, k}$ is $\lambda$-TP;

(iv) all triangular matrices $\left[L_{n, k}(f)\right]_{n, k},\left[\widetilde{L}_{n, k}(f)\right]_{n, k},\left[(-1)^{n-k} S_{n, k}(\bar{f}, 1)\right]_{n, k},\left[(-1)^{n-k} L_{n, k}(\bar{f})\right]_{n, k}$, and $\left[(-1)^{n-k} \widetilde{L}_{n, k}(\bar{f})\right]_{n, k}$ are TP;

(v) the A-convolution preserves the $S M$ property if $A$ is any of triangles $\left[S_{n, k}(f, g)\right]_{n, k}$ with $\lambda \geq 0,\left[S_{n+1, k+1}(f, 1)\right]_{n, k}$ and $\left[\widetilde{L}_{n, k}(f)\right]_{n, k}$;

(vi) the m-branched Stieltjes-type continued fractions

$$
\sum_{n \geq 0} S_{n}(f, g ; q) t^{n}=\frac{1}{1-\alpha_{m} t \prod_{i_{1}=1}^{m} \frac{1}{1-\alpha_{m+i_{1}} t \prod_{i_{2}=1}^{m} \frac{1}{1-\alpha_{m+i_{1}+i_{2}} t \prod_{i_{3}=1}^{m} \frac{1}{1-\cdots}}}}
$$

and

$$
\sum_{n \geq 0} S_{n}^{*}(f, g ; q) t^{n}=\frac{1}{1-\beta_{m} t \prod_{i_{1}=1}^{m} \frac{1}{1-\beta_{m+i_{1}} t \prod_{i_{2}=1}^{m} \frac{1}{1-\beta_{m+i_{1}+i_{2}} t \prod_{i_{3}=1}^{m} \frac{1}{1-\cdots}}}}
$$

with coefficients

$$
\begin{aligned}
& \left(\alpha_{i}\right)_{i \geq m}=(\lambda+q, \underbrace{x_{1}, \ldots, x_{m}}_{m}, \lambda+q, \underbrace{2 x_{1}, \ldots, 2 x_{m}}_{m}, \lambda+q, \underbrace{3 x_{1}, \ldots, 3 x_{m}}_{m}, \ldots) \\
& \left(\beta_{i}\right)_{i \geq m}=(q \lambda+1, \underbrace{q x_{1}, \ldots, q x_{m}}_{m}, q \lambda+1, \underbrace{2 q x_{1}, \ldots, 2 q x_{m}}_{m}, q \lambda+1, \underbrace{3 q x_{1}, \ldots, 3 q x_{m}}_{m}, \ldots)
\end{aligned}
$$

if $1 / \bar{f}^{\prime}(t)=\prod_{i=1}^{m}\left(1+x_{i} t\right)$.

Theorem 1.7. If $1 /\left[(1+t) \bar{f}^{\prime}(t)\right]$ is a PF function and $g(t)=(1+f(t))^{\gamma} \exp (\lambda f(t))$, then we have 
(i) both $\left(S_{n}(f, g ; q)\right)_{n \geq 0}$ and $\left(S_{n}^{*}(f, g ; q)\right)_{n \geq 0}$ are $(\gamma, \lambda, q)$-SM and 3 - $(\gamma, \lambda, q)$-log-convex;

(ii) $\left(S_{n}\left(f,(1+f)^{\gamma} ; q\right)\right)_{n \geq 0}$ is $(\gamma, q)-S M$ and $3-(\gamma, q)$-log-convex;

(iii) $\left(f_{n+1}\right)_{n \geq 0}$ is $S M$ and 3-log-convex;

(iv) $\left[S_{n, k}(f, g)\right]_{n, k}$ is $(\gamma, \lambda)-T P$;

(v) $\left[(-1)^{n-k} S_{n, k}(\bar{f}, 1 / g(\bar{f}))\right]_{n, k}$ is TP for $\gamma \geq 0$ and $\lambda \geq 0$;

(vi) the convolution $z_{n}=\sum_{k=0}^{n} S_{n, k}(f, g) x_{k} y_{n-k}$ preserves the $S M$ property for $\gamma \geq 0$ and $\lambda \geq 0$.

In the following, we will present the proof of Theorem 1.1 in Section 2. In Section 3, using the theory of exponential Riordan arrays, we will present some generalized results for total positivity. Then we use them to prove Theorems 1.2, 1.3, 1.6 and 1.7. In Section 4, we apply our results to some combinatorial triangles including Bessel triangles of two kinds and their generalizations, the Lah triangle and its generalization, the idempotent triangle and some triangles related to binomial coefficients, rook polynomials and Laguerre polynomials. We not only get total positivity of these lower-triangles, and $q$-Stieltjes moment properties and 3- $q$-log-convexity of their row-generating functions, but also prove that their triangular convolutions preserve Stieltjes moment property.

\section{Total positivity of the Toeplitz matrix}

The next result will be used repeatedly, which follows from the classical Cauchy-Binet formula.

Lemma 2.1. The product of two $\boldsymbol{x}_{-T P_{r}}\left(\boldsymbol{x}\right.$-TP, resp.) matrices is still $\boldsymbol{x}$ - $T P_{r}$ ( $\boldsymbol{x}$-TP, resp.).

The Proof of Theorem 1.1: Note for the convolution $c_{n}=\sum_{k \geq 0} a_{k} b_{n-k}$ for $n \geq 0$ that we have the decomposition

$$
\left[c_{i-j}\right]_{i, j \geq 0}=\left[a_{i-j}\right]_{i, j \geq 0}\left[b_{i-j}\right]_{i, j \geq 0}
$$

So, by Lemma 2.1, the product of two $\mathrm{PF}_{r}$ functions is a $\mathrm{PF}_{r}$ function. Since $g(t)$ is still a $\mathrm{PF}_{r}$, for the Pólya frequency of order $r$ of the product of $g(t)$ and $\exp \left(\sum_{j \geq 1} x_{j} \frac{t^{j}}{j}\right)$, it suffices to prove a stronger result that the Toeplitz matrix $\left[\mathcal{A}_{i-j}(\mathbf{x})\right]_{i, j \geq 0}$ is $\mathrm{TP}$ when $g(t)=1$.

By taking derivative in $t$ on both sides of the equality

$$
\sum_{n \geq 0} \mathcal{A}_{n}(\mathbf{x}) t^{n}=\exp \left(\sum_{j \geq 1} x_{j} \frac{t^{j}}{j}\right)
$$

we have

$$
\sum_{n \geq 1} n \mathcal{A}_{n}(\mathbf{x}) t^{n-1}=\sum_{j \geq 1} x_{j} t^{j-1} \sum_{n \geq 0} \mathcal{A}_{n}(\mathbf{x}) t^{n} .
$$


This implies for $n \geq 1$ that

$$
n \mathcal{A}_{n}(\mathbf{x})=\sum_{j=1}^{n} x_{j} \mathcal{A}_{n-j}(\mathbf{x}) .
$$

This recurrence relation is closely related to the complete symmetric function $h_{n}$ and the power sum symmetric function $p_{n}$, which satisfy

$$
n h_{n}=\sum_{j=1}^{n} p_{j} h_{n-j} .
$$

Moreover, it is also known that the ordinary generating function of $h_{n}$ can be written as

$$
\sum_{n \geq 0} h_{n} t^{n}=\prod_{i \geq 1} \frac{1}{1-\beta_{i} t} .
$$

So $\left(h_{n}\right)_{n \geq 0}$ forms a PF sequence for $\beta_{i} \geq 0$ for $i \geq 1$. In consequence, if there exists a sequence $\left(\lambda_{i}\right)_{i \geq 1}$ of nonnegative real numbers such that $x_{n}=\sum_{i \geq 1} \lambda_{i}^{n}$, then $\mathcal{A}_{n}(\mathbf{x})$ is a complete symmetric function of $\left(\lambda_{i}\right)_{i \geq 1}$. So $\left(\mathcal{A}_{n}(\mathbf{x})\right)_{n \geq 0}$ is a PF sequence. That is to say that the Toeplitz matrix $\left[\mathcal{A}_{i-j}(\mathbf{x})\right]_{i, j \geq 0}$ is TP. The proof is complete.

\section{Exponential Riordan arrays and total positivity}

\subsection{Definition and properties of exponential Riordan arrays}

The set of Sheffer polynomials forms a group called the Sheffer group, which is isomorphic to the exponential Riordan group. First let us recall some properties of the exponential Riordan array. An exponential Riordan array [4, 21, 22], denoted by $R=\left[R_{n, k}\right]_{n, k}=$ $(g(t), f(t))$, is an infinite lower triangular matrix whose exponential generating function of the $k$ th column is

$$
\frac{g(t) f^{k}(t)}{k !}
$$

for $k=0,1,2, \ldots$, where $g(0) f^{\prime}(0) \neq 0$ and $f(0)=0$. That is to say for $n, k \geq 0$ that

$$
R_{n, k}=\frac{n !}{k !}\left[t^{n}\right] g(t) f^{k}(t) .
$$

Let $R_{n}(q)=\sum_{k=0}^{n} R_{n, k} q^{k}$ be the row-generating function of $R$. Then we have

$$
\sum_{n \geq 0} R_{n}(q) \frac{t^{n}}{n !}=g(t) \exp (q f(t)) .
$$

The group law is then given by

$$
(g, f) *(h, \ell)=(g \times h(f), \ell(f)) .
$$

The identity for this law is $I=(1, t)$ and the inverse of $(g, f)$ is $(g, f)^{-1}=(1 /(g(\bar{f})), \bar{f})$, where $\bar{f}$ is the compositional inverse of $f$.

An exponential Riordan array can also be defined by the next recurrence relation. 
Proposition 3.1. [21] Let $\left[R_{n, k}\right]_{n, k \geq 0}=(g(t), f(t))$ be an exponential Riordan array. Then there exist two sequences $\left(z_{n}\right)_{n \geqslant 0}$ and $\left(a_{n}\right)_{n \geqslant 0}$ such that

$$
R_{0,0}=1, \quad R_{n, 0}=\sum_{i \geqslant 0} i ! z_{i} R_{n-1, i}, \quad R_{n, k}=\frac{1}{k !} \sum_{i \geqslant k-1} i !\left(z_{i-k}+k a_{i-k+1}\right) R_{n-1, i}
$$

for $n, k \geqslant 1$. In particular,

$$
Z(t)=\frac{g^{\prime}(\bar{f}(t))}{g(\bar{f}(t))}, \quad A(t)=f^{\prime}(\bar{f}(t)),
$$

where $Z(t)=\sum_{n \geqslant 0} z_{n} t^{n}$ and $A(t)=\sum_{n \geqslant 0} a_{n} t^{n}$.

The next result follows immediately from Proposition 3.1.

Proposition 3.2. Let $\left[R_{n, k}\right]_{n, k \geq 0}=(g(t), f(t))$ be an exponential Riordan array. Assume $\mathfrak{R}=\left[\mathfrak{R}_{n, k}\right]_{n, k}$, where $\mathfrak{R}_{n, k}=R_{n, k} k$ !. Then there exist two sequences $\left(z_{n}\right)_{n \geqslant 0}$ and $\left(a_{n}\right)_{n \geqslant 0}$ such that

$$
\mathfrak{R}_{0,0}=1, \quad \mathfrak{R}_{n, 0}=\sum_{i \geqslant 0} z_{i} \mathfrak{R}_{n-1, i}, \quad \mathfrak{R}_{n, k}=\sum_{i \geqslant k-1}\left(z_{i-k}+k a_{i-k+1}\right) \mathfrak{R}_{n-1, i}
$$

for $n, k \geqslant 1$, where

$$
Z(t)=\sum_{n \geqslant 0} z_{n} t^{n}=\frac{g^{\prime}(\bar{f}(t))}{g(\bar{f}(t))}, \quad A(t)=\sum_{n \geqslant 0} a_{n} t^{n}=f^{\prime}(\bar{f}(t)) .
$$

Associated to each exponential Riordan array $R=(g(t), f(t))$, there is a matrix $P=$ $\left(p_{i, j}\right)_{i, j \geqslant 0}$, called the production matrix, such that

$$
\bar{R}=R P,
$$

where $\bar{R}$ is obtained from $R$ with the first row removed. Assume that $z_{-1}=0$. Deutsch et al. [21] obtained the production matrix

$$
P=\left[p_{i, j}\right]_{i, j \geqslant 0}=\left[\begin{array}{ccccc}
z_{0} & a_{0} & & & \\
1 ! z_{1} & \frac{1 !}{1 !}\left(z_{0}+a_{1}\right) & a_{0} & & \\
2 ! z_{2} & \frac{2 !}{1 !}\left(z_{1}+a_{2}\right) & \frac{2 !}{2 !}\left(z_{0}+2 a_{1}\right) & a_{0} & \\
3 ! z_{3} & \frac{3 !}{1 !}\left(z_{2}+a_{3}\right) & \frac{3 !}{2 !}\left(z_{1}+2 a_{2}\right) & \frac{3 !}{3 !}\left(z_{0}+3 a_{1}\right) & \ddots \\
\vdots & \vdots & \vdots & \vdots & \ddots
\end{array}\right]
$$

where the elements

$$
p_{i, j}=\frac{i !}{j !}\left(z_{i-j}+j a_{i-j+1}\right)
$$

for $i, j \geqslant 0$.

Remark 3.3. By Proposition (3.2), for the array $\mathfrak{R}$ there is a matrix $\mathcal{P}=\left(\mathcal{P}_{i, j}\right)_{i, j \geqslant 0}$ with

$$
\mathcal{P}_{i, j}=z_{i-j}+j a_{i-j+1}
$$

for $i, j \geqslant 0$ such that

$$
\bar{\Re}=\mathfrak{R} \text {. }
$$




\subsection{Total positivity of exponential Riordan arrays}

The following result for total positivity is a special case of [40, Theorems 9.4, 9.7].

Lemma 3.4. [40] Let $M=\left[M_{n, k}\right]_{n, k \geq 0}$ be a lower triangular matrix with $M_{0,0}=1$ and $\bar{M}=M \mathcal{P}$. If $\mathcal{P}$ is $\boldsymbol{x}$-TP $P_{r}$, then both the matrix $\left[M_{n, k}\right]_{n, k \geq 0}$ and the Hankel matrix $\left[M_{i+j, 0}\right]_{i, j \geq 0}$ are $\boldsymbol{x}-T P_{r}$.

We will apply Lemma 3.4 to the exponential Riordan array $\left[R_{n, k}\right]_{n, k \geq 0}$ and its associated array $\left[\Re_{n, k}\right]_{n, k \geq 0}$. The following is obvious and we omit its proof for brevity.

Lemma 3.5. Let $c_{n}$ and $d_{n}$ be positive real numbers for $n \geq 0$. Then a matrix $M=$ $\left[M_{n, k}\right]_{n, k \geq 0}$ is $T P_{r}$ if and only if the matrix $M(\boldsymbol{c}, \boldsymbol{d})=\left[c_{n} d_{k} M_{n, k}\right]_{n, k \geq 0}$ is $T P_{r}$.

Theorem 3.6. Assume that $g(t)$ is a $P F_{r_{1}}$ function. If one of $f(t)$ and $1 / \bar{f}^{\prime}(t)$ is a $P F_{r_{2}}$, then exponential Riordan arrays $\left(g(t) e^{\lambda f(t)}, f(t)\right)$ and $\left(g(f(t)) e^{\lambda f(t)}, f(t)\right)$ are $\lambda$ $T P_{r}$, where $r=\min \left\{r_{1}, r_{2}\right\}$.

Proof. We first claim that the total positivity of order $r$ of $(g(t), f(t))$ implies the $\lambda$-total positivity of order $r$ of $\left(g(t) e^{\lambda f(t)}, f(t)\right)$. This can be proved as follows. Let $R=\left[R_{n, k}\right]_{n, k}=$ $(g(t), f(t))$ and $\widehat{R}=\left[\widehat{R}_{n, k}\right]_{n, k}=\left(g(t) e^{\lambda f(t)}, f(t)\right)$. Then

$$
\begin{aligned}
& \sum_{n} \sum_{k} R_{n, k} q^{k} \frac{t^{n}}{n !}=g(t) e^{q f(t)}, \\
& \sum_{n} \sum_{k} \widehat{R}_{n, k} q^{k} \frac{t^{n}}{n !}=g(t) e^{(q+\lambda) f(t)},
\end{aligned}
$$

which implies

$$
\widehat{R}_{n, k}=\sum_{i \geq k} R_{n, i}\left(\begin{array}{l}
i \\
k
\end{array}\right) \lambda^{i-k}
$$

Obviously,

$$
\widehat{R}=R B
$$

where $B=\left[\left(\begin{array}{l}n \\ k\end{array}\right) \lambda^{n-k}\right]_{n, k}$. From the total positivity of the Pascal triangle $\left[\left(\begin{array}{l}n \\ k\end{array}\right)\right]_{n, k}$, we immediately get that $B$ is $\lambda$-TP. Thus, by Lemma 2.1 , it follows from $\widehat{R}=R B$ that the total positivity of order $r$ of $R$ implies the $\lambda$-total positivity of order $r$ of $\widehat{R}$. Similarly, we can deduce that the total positivity of order $r$ of $(g(f(t)), f(t))$ implies the $\lambda$-total positivity of order $r$ of $\left(g(f(t)) e^{\lambda f(t)}, f(t)\right)$.

In what follows we only need to prove the total positivity of order $r$ of $(g(t), f(t))$ and $(g(f(t)), f(t))$.

In terms of the rule (3.2) about the product of Riordan arrays, we have decompositions

$$
\begin{aligned}
(g(t), f(t)) & =(g(t), t)(1, f(t)), \\
(g(f(t)), f(t)) & =(1, f(t))(g(t), t) .
\end{aligned}
$$

Thus, in order to demonstrate total positivity of $(g(t), f(t))$ and $(g(f(t)), f(t))$, in terms of Lemma 2.1, it suffices to prove that $(g(t), t)$ and $(1, f(t))$ are $\mathrm{TP}_{r}$. 
Let $G=\left[G_{n, k}\right]_{n, k \geq 0}$, where $G_{n, k}=\left[t^{n}\right] g(t) t^{k}$. It follows from Lemma 3.5 that the total positivity of $G$ implies that of $(g(t), t)$. Obviously, $G=\Gamma(g)$ is the Toeplitz matrix of the sequence $\left(g_{n}\right)_{n \geq 0}$, which is $\mathrm{TP}_{r}$.

In the following, we will prove that $(1, f(t))$ is $\operatorname{TP}_{r}$ if one of $f(t)$ and $1 / \bar{f}^{\prime}(t)$ is a Pólya frequency function of order $r_{2}$.

(i) Assume that $f(t)$ is a Pólya frequency function of order $r_{2}$. Let $F=\left[F_{n, k}\right]_{n, k \geq 0}$ with $F_{n, k}=\left[t^{n}\right] f^{k}(t)$. We give two different proofs as follows.

The first proof: It follows from Lemma 3.5 that the total positivity of $F$ implies that of $(1, f(t))$. Denote $F$ by $\left[1, f(x), f^{2}(x), f^{3}(x), \ldots\right]$ and its submatrix of the first $n$ columns by

$$
\left[1, f(x), f^{2}(x), f^{3}(x), f^{n-1}(x)\right]=\left[\begin{array}{c}
C_{n, n} \\
D_{\infty, n}
\end{array}\right] .
$$

Let $h(t)=f(t) / t=\sum_{n \geq 0} h_{n} t^{n}$ and $\Gamma(h)$ denote the Toeplitz matrix of $\left(h_{n}\right)_{n \geq 0}$. Then we have

$$
\left[\begin{array}{cc}
C_{n, n} & 0 \\
D_{\infty, n} & \Gamma\left(h^{n-1}\right)
\end{array}\right]\left[\begin{array}{cc}
I_{n} & 0 \\
0 & \Gamma(h)
\end{array}\right]=\left[\begin{array}{cc}
C_{n, n} & 0 \\
D_{\infty, n} & \Gamma\left(h^{n}\right)
\end{array}\right] .
$$

In addition, it is obvious that

$$
\left[\begin{array}{cc}
C_{n, n} & 0 \\
D_{\infty, n} & \Gamma\left(h^{n}\right)
\end{array}\right]=\left[\begin{array}{cc}
C_{n+1, n+1} & 0 \\
D_{\infty, n+1} & \Gamma\left(h^{n}\right)
\end{array}\right] .
$$

Thus, we get

$$
\left[\begin{array}{cc}
C_{n, n} & 0 \\
D_{\infty, n} & \Gamma\left(h^{n-1}\right)
\end{array}\right]\left[\begin{array}{cc}
I_{n} & 0 \\
0 & \Gamma(h)
\end{array}\right]=\left[\begin{array}{cc}
C_{n+1, n+1} & 0 \\
D_{\infty, n+1} & \Gamma\left(h^{n}\right)
\end{array}\right] .
$$

In order to prove total positivity of order $r_{2}$ of $F$, it suffices to show that of $\left[\begin{array}{c}C_{n, n} \\ D_{\infty, n}\end{array}\right]$ for all $n$. Note that $\left[\begin{array}{cc}I_{n} & 0 \\ 0 & \Gamma(h)\end{array}\right]$ and the Toeplitz matrix $\Gamma\left(h^{n}\right)$ are $\operatorname{TP}_{r_{2}}$ because $f(t)$ is a Pólya frequency function of order $r_{2}$. Applying Lemma 2.1 to (3.8), by induction on $n$, we have

$$
\left[\begin{array}{cc}
C_{n, n} & 0 \\
D_{\infty, n} & \Gamma\left(h^{n-1}\right)
\end{array}\right]
$$

is $\operatorname{TP}_{r_{2}}$ for all $n$. In particular, the submatrix $\left[\begin{array}{c}C_{n, n} \\ D_{\infty, n}\end{array}\right]$ is $\operatorname{TP}_{r_{2}}$ for all $n$. Thus $F$ is $\operatorname{TP}_{r_{2}}$.

The second proof: ${ }^{1}$ Note that $F_{n, k}=\left[t^{n}\right] f^{k}(t)=\left[t^{n}\right] f(t) f^{k-1}(t)$ for $k \geq 1$. Let $f(t)=\sum_{n \geq 0} f_{n} t^{n}$. Then we have

$$
F_{n, k}=\sum_{j=k}^{n} f_{n-j+1} F_{j-1, k-1}
$$

for $n, k \geq 1$. Let $\overrightarrow{F_{n}}$ denote the matrix consisting of columns from 1 to $n$ and $F_{n}$ denote the matrix consisting of columns from 0 to $n-1$ of $F$. It follows from (3.9) that

$$
\overrightarrow{F_{n}}=\Gamma(f(t) / t) F_{n} .
$$

\footnotetext{
1 This method is actually the same as that of [19] for total positivity, where it was given as a constructed method.
} 
Thus in terms of Lemma 2.1 and total positivity of $\Gamma(f)$, we obtain that $F_{n}$ is $\mathrm{TP}_{r_{2}}$ by induction on $n$. So the matrix $F$ is $\mathrm{TP}_{r_{2}}$.

(ii) Assume that $1 / \bar{f}^{\prime}(t)$ is a Pólya frequency function of order of $r_{2}$.

For the exponential Riordan array $(1, f(t))$, by Proposition 3.1, there exist two functions $Z(t)$ and $A(t)$ such that

$$
Z(t)=\frac{g^{\prime}(\bar{f}(t))}{g(\bar{f}(t))}=0, \quad A(t)=f^{\prime}(\bar{f}(t)) .
$$

On the other hand, it follows from $f(\bar{f}(t))=t$ that

$$
f^{\prime}(\bar{f}(t))=\frac{1}{\bar{f}^{\prime}(t)}
$$

where $\bar{f}^{\prime}(t)$ is the derivative function of $\bar{f}(t)$. Thus, we have

$$
A(t)=\frac{1}{\bar{f}^{\prime}(t)}
$$

In consequence, $A(t)$ is a Pólya frequency of order $r_{2}$ in terms of the Pólya frequency of order $r_{2}$ of $1 / \bar{f}^{\prime}(t)$ and (3.10). So the Toeplitz matrix $\Gamma(A)=\left[a_{i-j}\right]_{i, j \geq 0}$ is $\operatorname{TP}_{r_{2}}$.

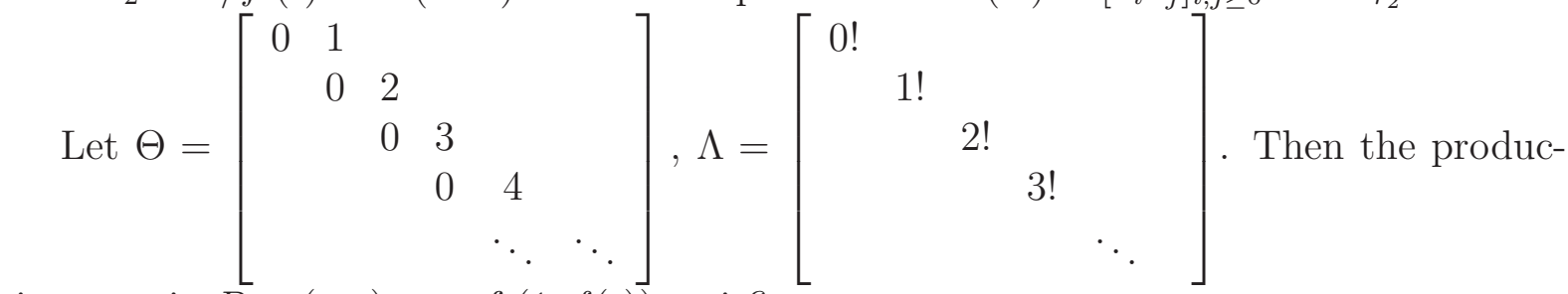
tion matrix $P=\left(p_{i, j}\right)_{i, j \geqslant 0}$ of $(1, f(t))$ satisfies

$$
p_{i, j}=\frac{i !}{j !} j a_{i-j+1},
$$

for $i, j \geqslant 0$, i.e.,

$$
P=\Lambda \Gamma(A) \Theta \Lambda^{-1}
$$

In consequence, by Lemma 3.5, we immediately get that the production matrix $P$ is $\mathrm{TP}_{r_{2}}$. Then by Lemma 3.4, we have the exponential Riordan array $(1, f(t))$ is $\mathrm{TP}_{r_{2}}$. This completes the proof.

Remark 3.7. For an exponential Riordan array $(g(t), f(t))$, it is often called a proper exponential Riordan array for $g(0) f^{\prime}(0) \neq 0$ and $f(0)=0$. If we drop the restricted condition, then it is called a general exponential Riordan array. From the proof of Theorem 3.6, it is obvious that the general exponential Riordan arrays $\left(g(t) e^{\lambda f(t)}, f(t)\right)$ and $\left(g(f(t)) e^{\lambda f(t)}, f(t)\right)$ are also $\lambda$ - $\mathrm{TP}_{r}$ if both $g(t)$ and $f(t)$ are $\mathrm{PF}_{r}$ functions.

Remark 3.8. We can repeatedly use Theorem 3.6 to get total positivity of more exponential Riordan arrays whose $f(t)$ and $g(t)$ are not both PF. 


\section{Proof of Theorem 1.2:}

(i) By (3.1), using the exponential Riordan array, we have

$$
\left[S_{n, k}(f, g)\right]_{n, k \geq 0}=(g(t), f(t)), \quad\left[S_{n, k}(f, g(f))\right]_{n, k \geq 0}=(g(f(t)), f(t)) .
$$

Thus by Theorem 3.6, we get that both $\left[S_{n, k}(f, g)\right]_{n, k \geq 0}$ and $\left[S_{n, k}(f, g(f))\right]_{n, k \geq 0}$ are $\mathrm{TP}_{r}$, where $r=\min \left\{r_{1}, r_{2}\right\}$.

(ii) By (1.6), we have $S_{n, k}(f, g)=n ! A_{n, k}(f, g)$ and $S_{n, k}(f, g(f))=n ! A_{n, k}(f, g(f))$. Then (ii) immediately follows from (i) and Lemma 3.5.

(iii) Using the property of the partial complete Bell polynomial [20, Theorem A, p.140], we get

$$
L_{n}(f ; q)=\sum_{k=1}^{n}(k-1) ! S_{n, k}(f, 1) q^{k} .
$$

So we have $L_{n, k}(f)=(k-1) ! S_{n, k}(f, 1)$. Then by Lemma 3.5 and (i), we immediately get that $\left[L_{n, k}(f)\right]_{n, k \geq 0}$ is $\mathrm{TP}_{r}$.

(iv) In what follows we will show that $\left[\widetilde{L}_{n, k}(f)\right]_{n, k \geq 0}$ is $\operatorname{TP}_{r}$.

By taking derivative in $q$ of

$$
\log (1-q f(t))=\sum_{n \geq 1}-L_{n}(f ; q) \frac{t^{n}}{n !}=\sum_{n \geq 1}-\left(\sum_{k=1}^{n} L_{n, k}(f) q^{k}\right) \frac{t^{n}}{n !}
$$

we get

$$
\frac{-f(t)}{1-q f(t)}=\sum_{n \geq 1}-L_{n}^{\prime}(f ; q) \frac{t^{n}}{n !}=\sum_{n \geq 1}-\left(\sum_{k=1}^{n} k L_{n, k}(f) q^{k-1}\right) \frac{t^{n}}{n !} .
$$

Then we have

$$
\frac{q f(t)}{1-q f(t)}=\sum_{n \geq 1} q L_{n}^{\prime}(f ; q) \frac{t^{n}}{n !}=\sum_{n \geq 1}\left(\sum_{k=1}^{n} k L_{n, k}(f) q^{k}\right) \frac{t^{n}}{n !} .
$$

This implies

$$
\frac{1}{1-q f(t)}=1+\sum_{n \geq 1} q L_{n}^{\prime}(f ; q) \frac{t^{n}}{n !}=1+\sum_{n \geq 1}\left(\sum_{k=1}^{n} k L_{n, k}(f) q^{k}\right) \frac{t^{n}}{n !} .
$$

Because we define

$$
\frac{1}{1-q f(t)}:=1+\sum_{n \geq 1} \widetilde{L}_{n}(f ; q) \frac{t^{n}}{n !}=1+\sum_{n \geq 1}\left(\sum_{k=1}^{n} \widetilde{L}_{n, k}(f) q^{k}\right) \frac{t^{n}}{n !},
$$

we have the relations

$$
\widetilde{L}_{n}(f ; q)=q L_{n}^{\prime}(f ; q), \quad \widetilde{L}_{n, k}(f)=k L_{n, k}(f)=k ! S_{n, k}(f, 1)
$$

for $n \geq 1$. In consequence, by Lemma 3.5 and (i), we immediately get that $\left[\widetilde{L}_{n, k}(f)\right]_{n, k>0}$ is $\mathrm{TP}_{r}$. This completes the proof of Theorem 1.2. 


\subsection{Total positivity of the Hankel matrix of the 0th column}

In what follows we will consider total positivity of the Hankel matrix of the 0th column from the exponential Riordan array.

Theorem 3.9. Let $\varphi(t)$ be a $P F_{r}$ function. For an exponential Riordan array $\left[R_{n, k}\right]_{n, k}$, let $Z(t)=(\nu+\omega t) \varphi(t)$ and $A(t)=\left(a+b t+c t^{2}\right) \varphi(t)$. Then we have the following results.

(i) If the tridiagonal matrix

$$
J=\left[\begin{array}{cccccc}
\nu & a & & & \\
\omega & \nu+b & 2 a & & \\
& \omega+c & \nu+2 b & 3 a & \\
& & \omega+2 c & \nu+3 b & 4 a & \\
& & & \ddots & \ddots & \ddots
\end{array}\right]
$$

is $(a, b, c, \nu, \omega)-T P_{r}$, then the lower-triangular matrix $\left[R_{n, k}\right]_{n, k}$ and the Hankel matrix $\left[R_{i+j, 0}\right]_{i, j \geq 0}$ are $(a, b, c, \nu, \omega)-T P_{r}$;

(ii) If $\{\nu, \omega, a, b, c\} \subseteq \mathbb{R}^{\geq 0}, \nu \geq w$ and $b \geq a+c$, then the lower-triangular matrix $\left[R_{n, k}\right]_{n, k}$ and the Hankel matrix $\left[R_{i+j, 0}\right]_{i, j \geq 0}$ are TP;

(iii) If $\{\nu, \omega, a, b, c\} \subseteq \mathbb{R}^{\geq 0}, \nu \geq a$ and $b \geq \max \{a+c, a+\omega\}$, then the lower-triangular matrix $\left[R_{n, k}\right]_{n, k}$ and the Hankel matrix $\left[R_{i+j, 0}\right]_{i, j \geq 0}$ are TP;

(iv) If $\omega=c=0$ and $r \rightarrow \infty$, then the lower-triangular matrix $\left[R_{n, k}\right]_{n, k}$ and the Hankel matrix $\left[R_{i+j, 0}\right]_{i, j \geq 0}$ are $(a, b, \nu)-T P$.

(v) Let $\varphi(t)=\prod_{i=1}^{m}\left(1+x_{i} t\right)$. If $\omega=c=0$ and $a=1$, then we have the $m$-branched Stieltjes-type continued fraction

$$
\sum_{n \geq 0} R_{n, 0} t^{n}=\frac{1}{1-\alpha_{m} t \prod_{i_{1}=1}^{m} \frac{1}{1-\alpha_{m+i_{1}} t \prod_{i_{2}=1}^{m} \frac{1}{1-\alpha_{m+i_{1}+i_{2}} t \prod_{i_{3}=1}^{m} \frac{1}{1-\cdots}}}}
$$

with coefficients

$$
\left(\alpha_{i}\right)_{i \geq m}=(\nu, \underbrace{x_{1}, \ldots, x_{m}}_{m}, \nu+b, \underbrace{2 x_{1}, \ldots, 2 x_{m}}_{m}, \nu+2 b, \underbrace{3 x_{1}, \ldots, 3 x_{m}}_{m}, \ldots) .
$$

Proof. (i) In terms of Lemma 3.4, it suffices to prove that the production matrix of $\left[R_{n, k}\right]_{n, k}$ is $(a, b, c, \nu, \omega)-\mathrm{TP}_{r}$. For the exponential Riordan array $\left[R_{n, k}\right]_{n, k}$ with

$$
Z(t)=(\nu+\omega t) \varphi(t), \quad A(t)=\left(a+b t+c t^{2}\right) \varphi(t),
$$


it follows from (3.3) that its production matrix

$$
\begin{aligned}
& P=\Lambda \Gamma(Z) \Lambda^{-1}+\Lambda \Gamma(A) \Theta \Lambda^{-1}
\end{aligned}
$$

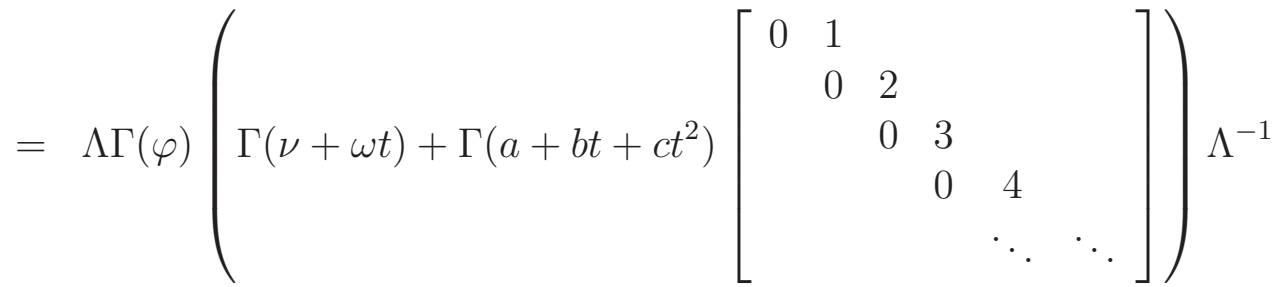

$$
\begin{aligned}
& =\Lambda \Gamma(\varphi)\left[\begin{array}{cccccc}
\nu & a & & & \\
\omega & \nu+b & 2 a & & \\
& \omega+c & \nu+2 b & 3 a & \\
& & \omega+2 c & \nu+3 b & 4 a & \\
& & & \ddots & \ddots & \ddots
\end{array}\right] \Lambda^{-1}
\end{aligned}
$$

Because the infinite Toeplitz matrix $\Gamma(\varphi)$ and the tridiagonal matrix $J$ are $(a, b, c, \nu, \omega)$ $\mathrm{TP}_{r}$, applying Lemma 2.1 to (3.17), we immediately have $P$ is $(a, b, c, \nu, \omega)-\mathrm{TP}_{r}$. This shows that (i) holds.

(ii) and (iii) are immediate from (i) because the conditions in (ii) and (iii) are sufficient to establish total positivity of $J$ (see $[17,73]$ for instance).

(iv) If $\omega=c=0$, then the tridiagonal matrix $J$ reduces to an upper-bidiagonal matrix

$$
\left[\begin{array}{cccccc}
\nu & a & & & & \\
& \nu+b & 2 a & & & \\
& & \nu+2 b & 3 a & & \\
& & \nu+3 b & 4 a & \\
& & & \ddots & \ddots & \ddots
\end{array}\right]
$$

which is $(a, b, \nu)$-TP. By (i), we immediately get that both the lower-triangular matrix $\left[R_{n, k}\right]_{n, k}$ and the Hankel matrix $\left[R_{i+j, 0}\right]_{i, j>0}$ are $(a, b, \nu)$-TP.

(v) Let $\varphi(t)=\prod_{i=1}^{m}\left(1+x_{i} t\right), \omega=c=0$ and $a=1$. Then we have

$$
\begin{aligned}
P & =\Lambda \Gamma(\varphi)\left[\begin{array}{cccccc}
\nu & a & & & \\
\omega & \nu+b & 2 a & & \\
& \omega+c & \nu+2 b & 3 a & & \\
& \omega+2 c & \nu+3 b & 4 a & \\
& & \ddots & \ddots & \ddots
\end{array}\right] \Lambda^{-1} \\
& =\left(\prod_{i=1}^{m} \Lambda \Gamma\left(1+x_{i} t\right) \Lambda^{-1}\right) \Lambda\left[\begin{array}{cccccc}
\nu & 1 & & & \\
& \nu+b & 2 & & & \\
& & \nu+2 b & 3 & & \\
& & & \nu+3 b & 4 & \\
& & & & \ddots & \ddots
\end{array}\right] \Lambda^{-1}
\end{aligned}
$$




$$
=\left(\prod_{i=1}^{m}\left[\begin{array}{ccccc}
1 & & & & \\
x_{i} & 1 & & & \\
& 2 x_{i} & 1 & & \\
& & 3 x_{i} & 1 & \\
& & & \ddots & \ddots
\end{array}\right]\right)\left[\begin{array}{cccccc}
\nu & 1 & & & & \\
& \nu+b & 1 & & & \\
& \nu+2 b & 1 & & \\
& & \nu+3 b & 1 & \\
& & & & \ddots & \ddots
\end{array}\right]
$$

which in terms of Proposition 7.2 and Proposition 8.2 (b) in [40] is exactly the production matrix for the $m$-branched Stieltjes-type continued fraction

$$
\sum_{n \geq 0} R_{n, 0} t^{n}=\frac{1}{1-\alpha_{m} t \prod_{i_{1}=1}^{m} \frac{1}{1-\alpha_{m+i_{1}} t \prod_{i_{2}=1}^{m} \frac{1}{1-\alpha_{m+i_{1}+i_{2}} t \prod_{i_{3}=1}^{m} \frac{1}{1-\cdots}}}}
$$

with coefficients $\left(\alpha_{i}\right)_{i \geq m}=(\nu, \underbrace{x_{1}, \ldots, x_{m}}_{m}, \nu+b, \underbrace{2 x_{1}, \ldots, 2 x_{m}}_{m}, \nu+2 b, \underbrace{3 x_{1}, \ldots, 3 x_{m}}_{m}, \ldots)$.

Remark 3.10. The $m$-branched Stieltjes-type continued fraction plays an important role in the coefficientwise Hankel-total positivity. It was shown that many combinatorial generating functions have $m$-branched Stieltjes-type continued fraction expansions. We refer the reader to [40] for more details.

Proposition 3.11. Let $A_{n}(q)$ be a polynomial of degree $n$. If $\left[A_{i+j}(q)\right]_{i, j}$ is $q$-TP , then so is $\left[A_{i+j}^{*}(q)\right]_{i, j}$.

Proof. For $k \leq r$, let $\mathcal{A}_{j_{1}, j_{2}, \ldots, j_{k}}^{i_{1}, i_{2}, \ldots, i_{k}}(q)$ (resp. $\left.\mathcal{B}_{j_{1}, j_{2}, \ldots, j_{k}}^{i_{1}, i_{2}, \ldots, i_{k}}(q)\right)$ be the minor of $\left[A_{i+j}(q)\right]_{i, j}$ (resp. $\left.\left[A_{i+j}^{*}(q)\right]_{i, j}\right)$ by taking its rows $i_{1}, i_{2}, \ldots, i_{k}$ and columns $j_{1}, j_{2}, \ldots, j_{k}$. In terms of the assumption that $\left[A_{i+j}(q)\right]_{i, j}$ is $q-\mathrm{TP}_{r}, \mathcal{A}_{j_{1}, j_{2}, \ldots, j_{k}}^{i_{1}, i_{2}, \ldots, i_{k}}(q)$ is a nonnegative coefficients polynomial of degree $\leq i_{1}+i_{2}+\cdots+i_{k}+j_{1}+j_{2}+\cdots+j_{k}$. Since $A_{i+j}^{*}(q)=q^{i+j} A_{i+j}(1 / q)$, taking out $q^{i_{1}}, q^{i_{2}}, \ldots, q^{i_{k}}$ from rows and $q^{j_{1}}, q^{j_{2}}, \ldots, q^{j_{k}}$ from columns of $\mathcal{B}_{j_{1}, j_{2}, \ldots, j_{k}}^{i_{1}, i_{2}, \ldots, i_{k}}(q)$, respectively, we get

$$
\mathcal{B}_{j_{1}, j_{2}, \ldots, j_{k}}^{i_{1}, i_{2}, \ldots, i_{k}}(q)=q^{i_{1}+i_{2}+\cdots+i_{k}+j_{1}+j_{2}+\cdots+j_{k}} \mathcal{A}_{j_{1}, j_{2}, \ldots, j_{k}}^{i_{1}, i_{2}, \ldots, i_{k}}(1 / q)
$$

In consequence, $\mathcal{B}_{j_{1}, j_{2}, \ldots, j_{k}}^{i_{1}, i_{2}, \ldots, i_{k}}(q)$ is a nonnegative coefficients polynomial. This completes the proof.

By Theorem 3.9 and Proposition 3.11, we get the next result, which in particular implies Theorem 1.3.

Theorem 3.12. Let $\left[E_{n, k}(f ; \lambda)\right]_{n, k \geq 0}=(\exp (\lambda f(t)), f(t))$ and $E_{n}(f ; \lambda, q)=\sum_{k \geq 0} E_{n, k}(f ; \lambda) q^{k}$. If $1 / \bar{f}^{\prime}(t)$ is a $P F_{r}$ function, then we have

(i) the Hankel matrices $\left[E_{i+j}(f ; \lambda, q)\right]_{i, j \geq 0}$ and $\left[E_{i+j}^{*}(f ; \lambda, q)\right]_{i, j \geq 0}$ are $(\lambda, q)-T P_{r}$;

(ii) the exponential Riordan array $\left[E_{n, k}(f ; \lambda)\right]_{n, k \geq 0}$ is $\lambda$-TP ;

(iii) the Hankel matrix $\left[f_{i+j+1}\right]_{i, j \geq 0}$ is $T P_{r}$; 
(iv) the exponential Riordan array $\left(f^{\prime}(x), f(x)\right)$ is $T P_{r}$;

(v) the row-generating function of $\left(f^{\prime}(x), f(x)\right)$ forms a q-Hankel-TP sequence.

Proof. For the exponential Riordan array $(\exp (\lambda f(t)), f(t))$, by (3.1), we have

$$
\sum_{n \geq 0} E_{n}(f ; \lambda, q) \frac{t^{n}}{n !}=\exp (\lambda f(t)) \exp (q f(t))=\exp ((\lambda+q) f(t)) .
$$

In order to prove that the Hankel matrix $\left[E_{i+j}(f ; \lambda, q)\right]_{i, j \geq 0}$ is $(\lambda, q)-\mathrm{TP}_{r}$, it suffices to prove that $\left[E_{i+j}(f ; \lambda, 0)\right]_{i, j \geq 0}$ is $\lambda-\mathrm{TP}_{r}$ by taking $\lambda \rightarrow \lambda+q$.

For the exponential Riordan array $(\exp (\lambda f(t)), f(t))$, by Proposition 3.1, there exist two functions $Z(t)$ and $A(t)$ such that

$$
Z(t)=\frac{\lambda}{\bar{f}^{\prime}(t)}, \quad A(t)=\frac{1}{\bar{f}^{\prime}(t)} .
$$

It follows from (iv) of Theorem 3.9 that $\left[E_{n, k}(f ; \lambda)\right]_{n, k \geq 0}$ and $\left[E_{i+j}(f ; \lambda, 0)\right]_{i, j \geq 0}$ are $\lambda$ $\mathrm{TP}_{r}$. Furthermore, it follows from $E_{n}^{*}(f ; \lambda, q)=q^{n} E_{n}(f ; \lambda, 1 / q)$ and Proposition 3.11 that $\left[E_{i+j}^{*}(f ; \lambda, q)\right]_{i, j \geq 0}$ is $(\lambda, q)-\mathrm{TP}_{r}$. This gives (i) and (ii).

By taking derivative in $t$ of

$$
\exp (\lambda f(t))=\sum_{n \geq 0} E_{n}(f ; \lambda, 0) \frac{t^{n}}{n !},
$$

we get

$$
\lambda f^{\prime}(t) \exp (\lambda f(t))=\sum_{n \geq 1} E_{n}(f ; \lambda, 0) \frac{t^{n-1}}{(n-1) !} .
$$

That is

$$
f^{\prime}(t) \exp (\lambda f(t))=\sum_{n \geq 0} \frac{E_{n+1}(f ; \lambda, 0)}{\lambda} \frac{t^{n}}{n !} .
$$

Since the Hankel matrix $\left[E_{i+j}(f ; \lambda, 0)\right]_{i, j \geq 0}$ is $\lambda-\mathrm{TP}_{r}$ and the constant term of $E_{n}(f ; \lambda, 0)$ is zero, $\left[\frac{E_{i+j+1}(f ; \lambda, 0)}{\lambda}\right]_{i, j \geq 0}$ is $\lambda$ - $\mathrm{TP}_{r}$. In particular, for $\lambda=0$, it reduces to that $\left[f_{i+j+1}\right]_{i, j \geq 0}$ is $\mathrm{TP}_{r}$ by (3.20). In addition, (3.20) implies

$$
\left(f^{\prime}(x), f(x)\right)=\left[E_{n+1, k+1}(f ; \lambda)\right]_{n, k \geq 0} .
$$

Then (iv) and (v) immediately follow from (i) and (ii). This completes the proof.

\section{The proof of Theorem 1.6:}

(i) and (ii) follow from Theorem 1.3 and Lemma 1.5.

For (iii), it follows from Theorem 3.6. Note a fact that if a matrix is TP then so is its singless inverse, see [41] for instance. Thus for (iv), it suffices to prove that both $\left[L_{n, k}(f)\right]_{n, k}$ and $\left[\widetilde{L}_{n, k}(f)\right]_{n, k}$ are TP, which follows from Theorem 1.2 (iii) and (iv). 
For (v), by (i) and Lemma 1.4, the convolution

$$
z_{n}=\sum_{k=0}^{n} S_{n, k}(f, g) x_{k} y_{n-k}
$$

preserves the SM property for $\lambda \geq 0$. Note that we have proved $\widetilde{L}_{n, k}(f)=k ! S_{n, k}(f, 1)$ in the proof of Theorem 1.2. Let $w_{k}=k ! x_{k}$, which is a Stieltjes moment sequence since the Hadamard product of two Stieltjes moment sequences is still a Stieltjes moment sequence. Then we get that the convolution

$$
z_{n}=\sum_{k=0}^{n} \widetilde{L}_{n, k}(f) x_{k} y_{n-k}=\sum_{k=0}^{n} S_{n, k}(f, 1) w_{k} y_{n-k}
$$

preserves the SM property. In addition, by Theorem 3.12 (v) and Lemma 1.4, we immediately get that the convolution

$$
z_{n}=\sum_{k=0}^{n} S_{n+1, k+1}(f, 1) x_{k} y_{n-k}
$$

also preserves the SM property.

Finally, for (vi), it follows from the proof of Theorem 3.12 that

$$
Z(t)=\frac{\lambda}{\bar{f}^{\prime}(t)}, \quad A(t)=\frac{1}{\bar{f}^{\prime}(t)}
$$

Then the first statement for $q=0$ of (vi) is immediate from Theorem $3.9(\mathrm{v})$. By taking $\lambda \rightarrow \lambda+q$, we get the first statement. Moreover, the second statement is from the first one by taking $q \rightarrow 1 / q$ and $t \rightarrow q t$. This completes the proof.

Theorem 3.13. Define an exponential Riordan array

$$
\left[R_{n, k}\right]_{n, k}=\left([f(t)+1]^{\gamma} \exp (\lambda f(t)), f(t)\right) .
$$

If $1 /\left[\bar{f}^{\prime}(t)(1+t)\right]$ is a $P F_{r}$ function, then we have

(i) the triangular matrix $\left[R_{n, k}\right]_{n, k}$ is $(\gamma, \lambda)-T P_{r}$;

(ii) the Hankel matrices $\left[T_{i+j}(q)\right]_{i, j \geq 0}$ and $\left[T_{i+j}^{*}(q)\right]_{i, j \geq 0}$ are $(\gamma, q)-T P_{r}$, where $\sum_{n \geq 0} T_{n}(q) \frac{t^{n}}{n !}=$ $[f(t)+1]^{\gamma} \exp (q f(t))$

(iii) the Hankel matrix $\left[f_{i+j+1}\right]_{i, j \geq 0}$ is $T P_{r}$;

(iv) the Hankel matrices $\left[R_{i+j}(x)\right]_{i, j \geq 0}$ and $\left[R_{i+j}^{*}(x)\right]_{i, j \geq 0}$ are $(\gamma, \lambda, x)-T P_{r}$, where $R_{n}(x)=$ $\sum_{k=0}^{n} R_{n, k} x^{k}$.

Proof. (i) Let $g(t)=[f(t)+1]^{\gamma} \exp (\lambda f(t))$. Then we derive

$$
\frac{g^{\prime}(t)}{g(t)}=\left(\frac{\gamma}{1+f(t)}+\lambda\right) f^{\prime}(t)
$$


For the exponential Riordan array $(g(t)), f(t))$, by Proposition 3.1, we derive

$$
Z(t)=\frac{g^{\prime}(\bar{f}(t))}{g(\bar{f}(t))}=\frac{(\gamma+\lambda+\lambda t) A(t)}{1+t}=\frac{(\gamma+\lambda+\lambda t)}{(1+t) \bar{f}^{\prime}(t)} .
$$

Let $\varphi(t)=\frac{1}{(1+t) \bar{f}^{\prime}(t)}$. So for the exponential Riordan array $\left.(g(t)), f(t)\right)$ we get

$$
Z(t)=(\gamma+\lambda+\lambda t) \varphi(t), \quad A(t)=(1+t) \varphi(t)
$$

It follows from [73, Proposition 3.3] that the tridiagonal matrix

$$
\left[\begin{array}{cccccc}
\gamma+\lambda & 1 & & & \\
\lambda & \gamma+\lambda+1 & 2 & & & \\
& \lambda & \gamma+\lambda+2 & 3 & & \\
& & \lambda & \gamma+\lambda+3 & 4 & \\
& & & \ddots & \ddots & \ddots
\end{array}\right]
$$

is $(\gamma, \lambda)$-TP. In consequence, by Theorem 3.9, both $\left[R_{n, k}\right]_{n, k}$ and $\left[T_{i+j}(\lambda)\right]_{i, j}$ are $(\gamma, \lambda)-\mathrm{TP}_{r}$. Obviously, Furthermore, by Proposition 3.11, we have $\left[T_{i+j}^{*}(\lambda)\right]_{i, j}$ is $(\gamma, \lambda)-\mathrm{TP}_{r}$. Thus, we show that (i) and (ii) hold.

(iii) By the hypothesis that $1 /\left[\bar{f}^{\prime}(t)(1+t)\right]$ is a $\mathrm{PF}_{r}$ function, $1 / \bar{f}^{\prime}(t)$ is a $\mathrm{PF}_{r}$ function. Then taking $\gamma=0$, we have the Hankel matrix $\left[f_{i+j+1}\right]_{i, j \geq 0}$ is $\mathrm{TP}_{r}$ by Theorem 3.12 (iii).

(iv) For the row-generating function $R_{n}(x)$, we have

$$
\sum_{n \geq 0} R_{n}(x) \frac{t^{n}}{n !}=[f(t)+1]^{\gamma} \exp ((\lambda+x) f(t)) .
$$

Thus we get $R_{n}(x)=T_{n}(\lambda+x)$ for $n \geq 0$. In consequence, (iv) is immediate from (ii).

Similar to the proof of Theorem 1.6, by Theorem 3.13, Lemma 1.5, and Lemma 1.4, we get Theorem 1.7.

\section{Applications}

Let $\left\langle\begin{array}{c}n \\ k\end{array}\right\rangle$ denote the Eulerian number, which counts the number of $n$-permutations with exactly $k-1$ excedances. It is well-known that it satisfies the recurrence relation

$$
\left\langle\begin{array}{l}
n \\
k
\end{array}\right\rangle=k\left\langle\begin{array}{c}
n-1 \\
k
\end{array}\right\rangle+(n-k+1)\left\langle\begin{array}{c}
n-1 \\
k-1
\end{array}\right\rangle
$$

with initial conditions $\left\langle\begin{array}{l}0 \\ 0\end{array}\right\rangle=1$ and $\left\langle\begin{array}{l}0 \\ k\end{array}\right\rangle=0$ for $k \geq 1$ or $k<0$. The triangular array $\left[\left\langle\begin{array}{c}n \\ k\end{array}\right\rangle\right]_{n, k \geq 0}$ is called the Eulerian triangle. Brenti proposed the next conjecture, which is still open. 
Conjecture 4.1. [11, Conjecture 6.10] The Eulerian triangle is TP.

Generally, let $\left[E_{n, k}\right]_{n, k \geq 0}$ be an array satisfying the recurrence relation:

$$
E_{n, k}=\left[a_{0} n+a_{1} k+a_{2}\right] E_{n-1, k}+\left[b_{0} n+b_{1} k+b_{2}\right] E_{n-1, k-1}
$$

with $E_{n, k}=0$ unless $0 \leq k \leq n$ and $E_{0,0}=1$. As we know that many classical enumerations satisfy the recurrence relation (4.1). In addition, some special cases of $\left[E_{n, k}\right]_{n, k \geq 0}$ are totally positive, for example, for $a_{0}=b_{0}=0$ or $a_{1}=b_{1}=0$, see Brenti [10]. In this section, we will present more results for total positivity of triangular arrays.

\subsection{A generalization of Bessel numbers of the second kind}

For $\{n, k\} \subseteq \mathbb{N}$, the Bessel number of the second kind $B_{n, k}$ is defined to be the number of partitions of $[n]:=\{1,2,3, \ldots, n\}$ into $k$ nonempty blocks of size at most 2 . It satisfies the recurrence relation

$$
B_{n, k}=B_{n-1, k-1}+(n-1) B_{n-2, k-1}
$$

with the initial condition $B_{0, k}=\delta_{0, k}$. We can get some properties of $B_{n, k}$ from the following more generalized recurrence relation for $a=1, b=\frac{1}{2}$ and $c=0$.

Proposition 4.2. Let $\{a, b, c\} \subseteq \mathbb{R}^{\geq 0}$. Assume that a triangular array $\left[\mathcal{B}_{n, k}\right]_{n, k \geq 0}$ satisfies the following recurrence relation

$$
\mathcal{B}_{n, k}=a \mathcal{B}_{n-1, k-1}+2 b(n-1) \mathcal{B}_{n-2, k-1}+3 c(n-1)(n-2) \mathcal{B}_{n-3, k-1}
$$

for $n, k \geq 1$, where $\mathcal{B}_{1,1}=1$ and $\mathcal{B}_{0, k}=\delta_{0, k}$. Then we have the following results.

(i) An explicit formula of $\mathcal{B}_{n, k}$ can be written as

$$
\mathcal{B}_{n, k}=\frac{n !}{k !} \sum_{i=0}^{k} a^{k-i} c^{n-k-i} b^{2 i-n+k}\left(\begin{array}{c}
k \\
i
\end{array}\right)\left(\begin{array}{c}
i \\
n-k-i
\end{array}\right)
$$

for $n, k \geq 1$.

(ii) The lower-triangular matrix $\left[\mathcal{B}_{n, k}\right]_{n, k \geq 0}$ is $T P_{r}$ for $b^{2} \geq 4 a c \cos ^{2} \frac{\pi}{r+1}$.

(iii) The lower-triangular matrix $\left[\mathcal{B}_{n, k}\right]_{n, k \geq 0}$ is TP for $b^{2} \geq 4 a c$.

Proof. Let $f_{k}(t)=\sum_{n \geq k} \mathcal{B}_{n, k} \frac{t^{n}}{n !}$. After we multiply $\frac{t^{n-1}}{(n-1) !}$ for both sides of (4.3) and sum on $n$, we get

$$
f_{k}^{\prime}(t)=\left(a+2 b t+3 c t^{2}\right) f_{k-1}(t)
$$

with $f_{0}(t)=1$ and $f_{k}(0)=0$ for $k \geq 1$. This implies that

$$
f_{k}(t)=\frac{\left(a t+b t^{2}+c t^{3}\right)^{k}}{k !}
$$

for $k \geq 1$. Thus the triangular array $\left[\mathcal{B}_{n, k}\right]_{n, k \geq 0}$ is the exponential Riordan array

$$
\left(1, a t+b t^{2}+c t^{3}\right)
$$


Then we immediately get

$$
\begin{aligned}
\mathcal{B}_{n, k} & =\frac{n !}{k !}\left[t^{n}\right] f^{k}(t) \\
& =\frac{n !}{k !}\left[t^{n-k}\right]\left(a+b t+c t^{2}\right)^{k} \\
& =\frac{n !}{k !}\left[t^{n-k}\right] \sum_{i=0}^{k}\left(\begin{array}{c}
k \\
i
\end{array}\right)\left(b t+c t^{2}\right)^{i} a^{k-i} \\
& =\frac{n !}{k !} \sum_{i=0}^{k}\left(\begin{array}{c}
k \\
i
\end{array}\right)\left[t^{n-k-i}\right](b+c t)^{i} a^{k-i} \\
& =\frac{n !}{k !} \sum_{i=0}^{k} a^{k-i} c^{n-k-i} b^{2 i-n+k}\left(\begin{array}{c}
k \\
i
\end{array}\right)\left(\begin{array}{c}
i \\
n-k-i
\end{array}\right)
\end{aligned}
$$

which is (i).

By Theorem 1.2, for (ii) and (iii), it suffices to prove that $a, b, c$ forms a $\mathrm{PF}_{r}$ sequence for $b^{2} \geq 4 a c \cos \frac{\pi}{r+1}$. This is true because its Toeplitz matrix is $\mathrm{TP}_{r}$ for $b^{2} \geq 4 a c \cos ^{2} \frac{\pi}{r+1}$, see [29].

Remark 4.3. A combinatorial interpretation of $\mathcal{B}_{n, k}$ can be given as follows: in any ordered partition of $[n]$, if a block only contains $n$ then we give it a weight $a$; if a block of size 2 contains $n$ then we give it a weight $b$; if a block of size 3 contains $n$ then we give it a weight $c$; for other block with weight 1 . Then

$$
\mathcal{B}_{n, k}=\sum_{\left\{\mathscr{B}_{1}, \mathscr{B}_{2}, \mathscr{B}_{3}, \ldots\right\}, 1 \leq\left|\mathscr{B}_{i}\right| \leq 3} \prod_{i} w\left(\mathscr{B}_{i}\right),
$$

where $\left\{\mathscr{B}_{1}, \mathscr{B}_{2}, \ldots \mathscr{B}_{k}\right\}$ is a partition of $[n]$ into $k$ nonempty ordered blocks of size at most 3 .

\subsection{A generalization of Bessel numbers of the first kind}

The famous Bessel polynomial introduced by Krall and Frink [32] has the following formula

$$
y_{n}(q)=\sum_{k=0}^{n} \frac{(n+k) !}{(n-k) ! k !}\left(\frac{q}{2}\right)^{k},
$$

which satisfies a second-order differential equation

$$
q^{2} y_{n}^{\prime \prime}+(2 q+2) y_{n}^{\prime}-n(n+1) y_{n}=0
$$

and the recurrence relation

$$
y_{n+1}(q)=(2 n+1) q y_{n}(q)+y_{n-1}(q)
$$

with initial conditions $y_{0}(q)=1$ and $y_{1}(q)=1+q$. It is well-known that Bessel polynomials form an orthogonal sequence of polynomials. See [15, 24] for more properties of Bessel polynomials. If we write

$$
y_{n-1}=\sum_{k \geq 0} b_{n, k} q^{n-k},
$$


then $b_{n, k}$ is called the signless Bessel number of the first kind. Denote the reverse Bessel polynomial by $Y_{n}(q)=\sum_{k \geq 0} b_{n, k} q^{k}$.

The reverse Bessel polynomial and the signless Bessel number satisfy

$$
\exp (q(1-\sqrt{1-2 t}))=\sum_{n \geq 0} Y_{n}(q) \frac{t^{n}}{n !}=\sum_{n \geq 0} \sum_{k=0}^{n} b_{n, k} q^{k} \frac{t^{n}}{n} .
$$

Note that $\left[b_{n, k}\right]_{n, k}$ satisfies the following recurrence relation:

$$
b_{n, k}=(2 n-k-2) b_{n-1, k}+b_{n-1, k-1}
$$

with $b_{0,0}=1$. Generalizing the recurrence relation (4.5), we define a generalized Bessel triangle $\left[\mathcal{T}_{n, k}\right]_{n, k \geq 0}$ and get some general results as follows, which in particular implies properties of the signless Bessel triangle $\left[b_{n, k}\right]_{n, k \geq 0}$ and the reverse Bessel polynomial $Y_{n}(q)$ for $a=2, b=c=1$ and $\lambda=d=0$.

Proposition 4.4. Let $a \in \mathbb{N}^{+}$, ad $\in \mathbb{N}$ and $c \in \mathbb{R}^{+}$. Assume that a generalized Bessel triangle $\left[\mathcal{T}_{n, k}\right]_{n, k \geq 0}$ satisfies the following recurrence relation

$$
\mathcal{T}_{n, k}=c \mathcal{T}_{n-1, k-1}+[a b(n-1)-b k+a b d+c \lambda] \mathcal{T}_{n-1, k}-b \lambda(k+1) \mathcal{T}_{n-1, k+1}
$$

for $n, k \geq 1$, where $\mathcal{T}_{0,0}=1$. Let $\mathcal{T}_{n}(q)=\sum_{k \geq 0} \mathcal{T}_{n, k} q^{k}$. Then we have the following results.

(i) An explicit formula of $\mathcal{T}_{n, k}$ can be written as

$$
\mathcal{T}_{n, k}=\frac{a^{n}}{k !} \sum_{j \geq 0} \sum_{i=0}^{k+j} \frac{(-1)^{n-i} b^{n-k-j} c^{k+j} \lambda^{j}}{j !}\left(\begin{array}{c}
k+j \\
i
\end{array}\right)\left(\frac{i-a d}{a}\right)_{n}
$$

for $n, k \geq 1$.

(ii) The lower-triangular matrix $\left[\mathcal{T}_{n, k}\right]_{n, k \geq 0}$ is $(b, \lambda)$-TP.

(iii) For $0 \leq a d \leq a-1,\left(\mathcal{T}_{n}(q)\right)_{n \geq 0}$ is $(b, \lambda, q)$-SM and 3 - $(b, \lambda, q)$-log-convex. In particular, $\left(\mathcal{T}_{n, 0}\right)_{n \geq 0}$ is $(b, \lambda)-S M$ and $3-(b, \lambda)$-log-convex.

(iv) The sequence $\left(f_{n}\right)_{n \geq 1}$ is $S M$, where $\sum_{n \geq 1} f_{n} \frac{t^{n}}{n !}=\frac{c}{b}\left[1-(1-a b t)^{\frac{1}{a}}\right]$ and $b>0$.

(v) If $0 \leq a d \leq a-1$ and $\{b, \lambda\} \subseteq \mathbb{R}^{\geq 0}$, then $z_{n}=\sum_{k \geq 0} \mathcal{T}_{n, k} x_{k} y_{n-k}$ preserves Stieltjes moment property of sequences.

Proof. Let $f_{k}(t)=\sum_{n \geq k} \mathcal{T}_{n, k} \frac{t^{n}}{n !}$. It follows from the recurrence relation (4.6) that

$$
f_{k}^{\prime}(t)=a b t f_{k}^{\prime}(t)+[b(a d-k)+c \lambda] f_{k}(t)+c f_{k-1}(t)-b \lambda(k+1) f_{k+1}
$$

for $k \geq 1$. This implies

$$
b \lambda(k+1) f_{k+1}+(1-a b t) f_{k}^{\prime}(t)-c f_{k-1}(t)+[b(k-a d)-c \lambda] f_{k}(t)=0
$$

for $k \geq 1$. It is not hard to check that

$$
f_{k}(t)=\frac{1}{k !}(1-a b t)^{-d} e^{\frac{c \lambda}{b}\left[1-(1-a b t)^{\frac{1}{a}}\right]}\left(\frac{c}{b}\right)^{k}\left[1-(1-a b t)^{\frac{1}{a}}\right]^{k}
$$


is the solution of equation (4.8). Let

$$
g(t)=(1-a b t)^{-d}, \quad f(t)=\frac{c}{b}\left[1-(1-a b t)^{\frac{1}{a}}\right] .
$$

Then we have $\left[\mathcal{T}_{n, k}\right]_{n, k}$ is the exponential Riordan array $\left(g(t) e^{\lambda f(t)}, f(t)\right)$. So by taking the coefficients of $t^{n}$ in $g(t) e^{\lambda f(t)} f(t)^{k}$, we immediately get

$$
\begin{aligned}
\mathcal{T}_{n, k} & =\frac{n !}{k !}\left[t^{n}\right] g(t) e^{\lambda f(t)} f(t)^{k} \\
& =\frac{n !}{k !}\left[t^{n}\right] g(t) \sum_{j \geq 0} \frac{\lambda^{j} f^{j+k}(t)}{j !} \\
& =\left(\frac{c}{b}\right)^{k} \frac{n !}{k !}\left[t^{n}\right](1-a b t)^{-d} \sum_{j \geq 0} \frac{\left(\frac{c \lambda}{b}\right)^{j}}{j !} \sum_{i=0}^{j+k}(-1)^{i}\left(\begin{array}{c}
j+k \\
i
\end{array}\right)(1-a b t)^{\frac{i}{a}} \\
& =\left(\frac{c}{b}\right)^{k} \frac{n !}{k !}\left[t^{n}\right] \sum_{j \geq 0} \sum_{i=0}^{k+j}(-1)^{i} \frac{\left(\frac{c \lambda}{b}\right)^{j}}{j !}\left(\begin{array}{c}
k+j \\
i
\end{array}\right)(1-a b t)^{\frac{i-a d}{a}} \\
& =\left(\frac{c}{b}\right)^{k} \frac{n !}{k !} \sum_{j \geq 0} \sum_{i=0}^{k+j}(-1)^{i} \frac{\left(\frac{c \lambda}{b}\right)^{j}}{j !}\left(\begin{array}{c}
k+j \\
i
\end{array}\right)(-a b)^{n} \frac{\left(\frac{i-a d}{a}\right)_{n}}{n !} \\
& =\frac{a^{n}}{k !} \sum_{j \geq 0} \sum_{i=0}^{k+j} \frac{(-1)^{n-i} b^{n-k-j} c^{k+j} \lambda^{j}}{j !}\left(\begin{array}{c}
k+j \\
i
\end{array}\right)\left(\frac{i-a d}{a}\right)_{n},
\end{aligned}
$$

which is (i).

Note that the compositional inverse of $f(t)$ is

$$
\bar{f}(t)=\frac{1-\left(1-\frac{b}{c} t\right)^{a}}{a b} .
$$

Clearly, both $g(t)$ and $1 / \bar{f}^{\prime}(t)=\frac{c}{\left(1-\frac{b}{c} t\right)^{a-1}}$ are Pólya frequency functions for $a \in \mathbb{N}^{+}, d \in \mathbb{N}$ and $c \in \mathbb{R}^{+}$. Then (ii) follows from Theorem 3.6.

For $d=0$, by Theorem 3.12 (i) and (iii), we obtain that $\left(\mathcal{T}_{n}(q)\right)_{n \geq 0}$ is $(\lambda, q)$-SM and $\left(f_{n}\right)_{n \geq 1}$ is SM. Furthermore, $\left(\mathcal{T}_{n}(q)\right)_{n \geq 0}$ is 3 - $(\lambda, q)$-log-convex and $z_{n}=\sum_{k \geq 0} \mathcal{T}_{n, k} x_{k} y_{n-k}$ preserves Stieltjes moment property of sequences by Lemmas 1.5 and 1.4, respectively.

For the general case $d \neq 0$, the corresponding total positivity in (iii) can be proved from the following production matrix. In addition, for the exponential Riordan array $\left(g(t) e^{\lambda f(t)}, f(t)\right)$, by $(3.1)$, we have

$$
\sum_{n \geq 0} \mathcal{T}_{n}(q) \frac{t^{n}}{n !}=g(t) \exp (\lambda f(t)) \exp (q f(t))=g(t) \exp ((\lambda+q) f(t)) .
$$

Thus, in order to prove that the Hankel matrix $\left[\mathcal{T}_{i+j}(q)\right]_{i, j \geq 0}$ is $(b, \lambda, q)$-TP, it suffices to prove that $\left[\mathcal{T}_{i+j}(0)\right]_{i, j \geq 0}$ is $(b, \lambda)$-TP, i.e., $\left[\mathcal{T}_{i+j, 0}\right]_{i, j \geq 0}$ is $(b, \lambda)$-TP.

For $Z(t)$ and $A(z)$ of the exponential Riordan array $\left(g(t) e^{\lambda f(t)}, f(t)\right)$, we derive

$$
Z(t)=\frac{a b d}{\left(1-\frac{b}{c} t\right)^{a}}+\frac{\lambda c}{\left(1-\frac{b}{c} t\right)^{a-1}}, A(t)=\frac{c}{\left(1-\frac{b}{c} t\right)^{a-1}} .
$$


We assume $p=a d \in[0, a-1]$. Then let $\varphi(t)=\frac{1}{\left(1-\frac{b}{c} t\right)^{a-p-1}}$ and $\phi(t)=\frac{c}{\left(1-\frac{b}{c} t\right)^{p}}$. We deduce the production matrix $P$ of the exponential Riordan array $\left(g(t) e^{\lambda f(t)}, f(t)\right)$ as follows:

$$
\begin{aligned}
& P=\Lambda \Gamma(Z) \Lambda^{-1}+\Lambda \Gamma(A) \Theta \Lambda^{-1} \\
& =\Lambda \Gamma(\varphi)\left(\Gamma\left(\frac{a b d}{\left(1-\frac{b}{c} t\right)^{p+1}}+\lambda \phi(t)\right)+\Gamma(\phi(t)) \Theta\right) \Lambda^{-1}
\end{aligned}
$$

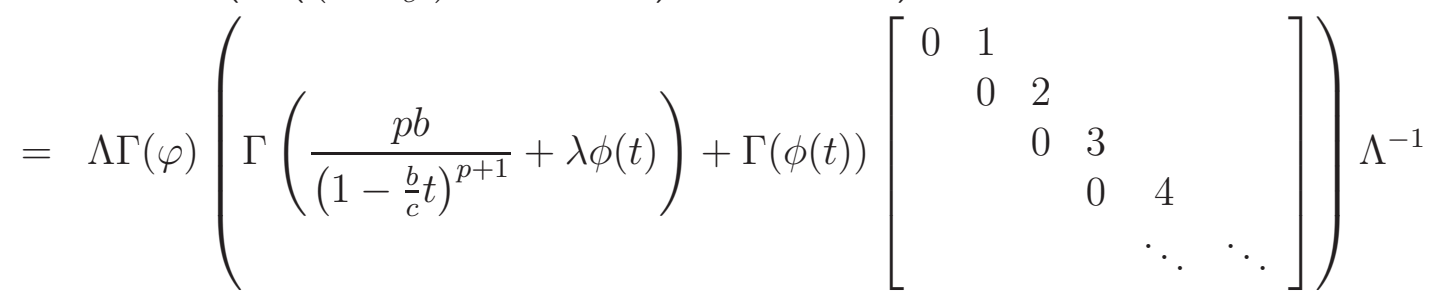

$$
\begin{aligned}
& =\Lambda \Gamma(\varphi)\left[c(i+1)\left(\begin{array}{l}
p+i-j \\
i-j+1
\end{array}\right)\left(\frac{b}{c}\right)^{i-j+1}+\lambda c\left(\begin{array}{c}
p-1+i-j \\
i-j
\end{array}\right)\left(\frac{b}{c}\right)^{i-j}\right]_{i, j} \Lambda^{-1} \\
& =\Lambda \Gamma(\varphi)\left[\begin{array}{cccccc}
\lambda c & c & & & \\
& \lambda c & 2 c & & \\
& & \lambda c & 3 c & & \\
& & & \lambda c & 4 c & \\
& & & & \ddots & \ddots
\end{array}\right] \Gamma(\phi) \Lambda^{-1}
\end{aligned}
$$

which is $(b, \lambda)$-TP. It follows from Theorem 3.9 (i) that we get the $(b, \lambda)$-total-positivity of $\left[\mathcal{T}_{n, k}\right]_{n \geq 0}$ in (ii), $(b, \lambda, q)$-Stieltjes moment property of $\left(\mathcal{T}_{n}(q)\right)_{n \geq 0}$, and $(b, \lambda)$-Stieltjes moment property of $\left(\mathcal{T}_{n, 0}\right)_{n \geq 0}$ in (iii). Furthermore, the remaining results in (iii) and (v) are immediate by Lemma 1.5 and Lemma 1.4, respectively.

Let $\left[\mathcal{T}_{n, k}\right]_{n, k}$ be defined by (4.6). For the reciprocal generalized Bessel polynomial $\mathcal{T}_{n}^{*}(q)=q^{n} \mathcal{T}_{n}(1 / q)$ and the reciprocal generalized Bessel numbers $\mathcal{T}_{n, k}^{*}=\mathcal{T}_{n, n-k}$, by Proposition 4.4 , we immediately get the following reciprocal result.

Proposition 4.5. Let $a \in \mathbb{N}^{+}$, ad $\in \mathbb{N}$ and $c \in \mathbb{R}^{+}$. If $0 \leq a d \leq a-1$, then we have

(i) the reciprocal generalized Bessel triangle $\left[\mathcal{T}_{n, k}^{*}\right]_{n, k \geq 0}$ satisfies the following recurrence $\mathcal{T}_{n, k}^{*}=c \mathcal{T}_{n-1, k}^{*}+[a b(n-1)-b(n-k)+a b d+c \lambda] \mathcal{T}_{n-1, k-1}^{*}-b \lambda(n-k+1) \mathcal{T}_{n-1, k-2}^{*}$ for $n, k \geq 1$, where $\mathcal{T}_{0,0}^{*}=1$;

(ii) the exponential generating function of $\mathcal{T}_{n}^{*}(q)$ can be written as

$$
\sum_{n \geq 0} \mathcal{T}_{n}^{*}(q) \frac{t^{n}}{n !}=g(q t) \exp ((q \lambda+1) f(q t) / q)
$$

(iii) $\left(\mathcal{T}_{n}^{*}(q)\right)_{n \geq 0}$ is $(b, \lambda, q)$-SM and 3 - $(b, \lambda, q)$-log-convex. In particular, $\left(\mathcal{T}_{n, n}^{*}\right)_{n \geq 0}$ is $(b, \lambda)$ $S M$ and $3-(b, \lambda)$-log-convex.

(iv) the convolution $z_{n}=\sum_{k \geq 0} \mathcal{T}_{n, k}^{*} x_{k} y_{n-k}$ preserves Stieltjes moment property of sequences for $\{b, \lambda\} \subseteq \mathbb{R}^{\geq 0}$. 
In [13], Callan proved

$$
\sum_{k=0}^{n} k !\left(\begin{array}{c}
2 n-k-1 \\
k-1
\end{array}\right)(2 n-2 k-1) ! !=(2 n-1) ! !
$$

which in fact counts different combinatorial structures, such as increasing ordered trees of $n$ edges by outdegree $k$ of the root. Let

$$
H_{n, k}=k !\left(\begin{array}{c}
2 n-k-1 \\
k-1
\end{array}\right)(2 n-2 k-1) ! !
$$

which is the sum of the weights of all vertices labeled $k$ at depth $n$ in the Catalan tree for $1 \leq k \leq n+1$ and $n \geq 0$, see [55, A102625]. In addition, $H_{n, k}$ satisfies the recurrence relation

$$
H_{n, k}=(2 n-k-2) H_{n-1, k}+k H_{n-1, k-1}
$$

with initial condition $H_{0,0}=1$. In fact, $H_{n, k}$ is closely related to the signless Bessel number $b_{n, k}$ by $H_{n, k}=b_{n, k} k$ !. Generalizing this relation (4.12), we consider the following triangle $\left[\widetilde{\mathcal{T}}_{n, k}\right]_{n, k \geq 0}$ related to the generalized Bessel triangle in Proposition (4.4) by $\widetilde{\mathcal{T}}_{n, k}=\mathcal{T}_{n, k} k$ ! Thus, by Theorem 1.6 and Proposition 4.4, we have the next result.

Proposition 4.6. Let $a \in \mathbb{N}^{+}$, ad $\in \mathbb{N}$ and $c \in \mathbb{R}^{+}$. Assume that a triangular array $\left[\widetilde{\mathcal{T}}_{n, k}\right]_{n, k \geq 0}$ satisfies the following recurrence relation

$$
\widetilde{\mathcal{T}}_{n, k}=c k \widetilde{\mathcal{T}}_{n-1, k-1}+[a b(n-1)-b k+a b d+c \lambda] \widetilde{\mathcal{T}}_{n-1, k}-b \lambda \widetilde{\mathcal{T}}_{n-1, k+1}
$$

for $n, k \geq 1$, where $\widetilde{\mathcal{T}}_{0,0}=1$. Let $\widetilde{\mathcal{T}}_{n}(q)=\sum_{k \geq 0} \widetilde{\mathcal{T}}_{n, k} q^{k}$. Then we have the following results.

(i) An explicit formula of $\widetilde{\mathcal{T}}_{n, k}$ can be written as

$$
\widetilde{\mathcal{T}}_{n, k}=a^{n} \sum_{j \geq 0} \sum_{i=0}^{k+j} \frac{(-1)^{n-i} b^{n-k-j} c^{k+j} \lambda^{j}}{j !}\left(\begin{array}{c}
k+j \\
i
\end{array}\right)\left(\frac{i-a d}{a}\right)_{n}
$$

for $n, k \geq 1$.

(ii) The lower-triangular matrix $\left[\widetilde{\mathcal{T}}_{n, k}\right]_{n, k \geq 0}$ is $(b, \lambda)-T P$.

(iii) For $d=0$, the exponential generating function is

$$
1+\sum_{n \geq 1} \widetilde{\mathcal{T}}_{n}(q) \frac{t^{n}}{n !}=\frac{1}{1-\frac{c}{b}\left[1-(1-a b t)^{\frac{1}{a}}\right](q+\lambda)}
$$

(iv) If $0 \leq a d \leq a-1$ and $\{b, \lambda\} \subseteq \mathbb{R}^{\geq 0}$, then the convolution $z_{n}=\sum_{k \geq 0} \widetilde{\mathcal{T}}_{n, k} x_{k} y_{n-k}$ preserves Stieltjes moment property of sequences. 


\subsection{A generalization of Lah numbers}

It is well known that the exponential partial Bell polynomial $\mathbf{B}_{n, k}\left(x_{1}, x_{2}, \ldots, x_{n-k+1}\right)$ is defined by the series expansion:

$$
\sum_{n \geq 0} \mathbf{B}_{n}(\mathbf{x} ; q) \frac{t^{n}}{n !}=\exp \left(q \sum_{j \geq 1} x_{j} \frac{t^{j}}{j !}\right)
$$

where

$$
\mathbf{B}_{n}(\mathbf{x} ; q)=\sum_{k=1}^{n} \mathbf{B}_{n, k}\left(x_{1}, \ldots, x_{n-k+1}\right) q^{k} \quad \text { for } n \geq 1
$$

and $\mathbf{B}_{0}(\mathbf{x} ; q)=1$. For $q=1, \mathbf{B}_{n}(\mathbf{x} ; 1)$ is called the exponential complete Bell polynomial. See Comtet [20, p.133-134].

Note that the well-known signless Lah number

$$
\mathcal{L}_{n, k}=\mathbf{B}_{n, k}(1 !, 2 !, 3 !, \ldots)=\left(\begin{array}{c}
n-1 \\
k-1
\end{array}\right) \frac{n !}{k !}
$$

which satisfies

$$
\exp \left(\frac{q t}{1-t}\right)=\sum_{n \geq 0} \mathcal{L}_{n}(q) \frac{t^{n}}{n !}=\sum_{n \geq 0} \sum_{k=0}^{n} \mathcal{L}_{n, k} q^{k} \frac{t^{n}}{n}
$$

see $[20$, p.133-134]. It counts the number of partitions of $[n]$ into $k$ lists, where a list means an ordered subset [55, A008297]. In addition, the row-generating function

$$
\mathcal{L}_{n}(q)=\sum_{k=0}^{n} \mathcal{L}_{n, k} q^{k}
$$

is called the Lah polynomial. Notice that $\left[\mathcal{L}_{n, k}\right]_{n, k}$ satisfies the following recurrence relation:

$$
\mathcal{L}_{n, k}=(n+k-1) \mathcal{L}_{n-1, k}+\mathcal{L}_{n-1, k-1}
$$

for $n, k \geq 1$ with $\mathcal{L}_{0,0}=1$. Generalizing the recurrence relation (4.16), we give the following result for a generalized Lah triangle $\left[T_{n, k}\right]_{n, k \geq 0}$, which reduces to those of the signless Lah triangle $\left[\mathcal{L}_{n, k}\right]_{n, k \geq 0}$ for $a=b=c=1$ and $d=\lambda=0$.

Proposition 4.7. Let $a \in \mathbb{N}$. Assume that a generalized Lah triangle $\left[T_{n, k}\right]_{n, k \geq 0}$ satisfies the following recurrence relation

$$
T_{n, k}=c T_{n-1, k-1}+[a b(n-1)+b k+a b d+c \lambda] T_{n-1, k}+b \lambda(k+1) T_{n-1, k+1}
$$

for $n, k \geq 1$, where $T_{0,0}=1$. Let $T_{n}(q)=\sum_{k \geq 0} T_{n, k} q^{k}$. Then we have the following results.

(i) An explicit formula of $T_{n, k}$ can be given by

$$
T_{n, k}=\frac{a^{n}}{k !} \sum_{j \geq 0} \sum_{i=0}^{k+j} \frac{(-1)^{n+k+j-i} b^{n-k-j} c^{k+j} \lambda^{j}}{j !}\left(\begin{array}{c}
k+j \\
i
\end{array}\right)\left(-\frac{i+a d}{a}\right)_{n}
$$

for $n, k \geq 1$. 
(ii) For $d \in \mathbb{N}$ and $c \in \mathbb{R}^{+}$, the lower-triangular matrix $\left[T_{n, k}\right]_{n \geq 0}$ is $(b, \lambda)$-TP.

(iii) For $c=b$, the lower-triangular matrix $\left[T_{n, k}\right]_{n \geq 0}$ is $(b, d, \lambda)-T P$.

(iv) For $c=b$, the sequence $\left(T_{n}(q)\right)_{n \geq 0}$ is $(b, d, \lambda, q)$-SM and 3 -(b, $\left.d, \lambda, q\right)$-log-convex. In particular, $\left(T_{n, 0}\right)_{n \geq 0}$ is $(b, d, \lambda)-S M$ and $3-(b, d, \lambda)$-log-convex.

(v) For $b>0$, the sequence $\left(f_{n}\right)_{n \geq 1}$ is $S M$, where $\sum_{n \geq 1} f_{n} \frac{t^{n}}{n !}=\frac{(1-a b t)^{-\frac{1}{a}}-1}{b}$.

(vi) Let $c=b$. For $\{b, d, \lambda\} \subseteq \mathbb{R}^{\geq 0}$, the convolution $z_{n}=\sum_{k \geq 0} T_{n, k} x_{k} y_{n-k}$ preserves Stieltjes moment property of sequences.

(vii) Let $a+1=m$ and $d=0$. We have the $m$-branched Stieltjes-type continued fraction expression

$$
\sum_{n \geq 0} T_{n}(q) t^{n}=\frac{1}{1-\alpha_{m} t \prod_{i_{1}=1}^{m} \frac{1}{1-\alpha_{m+i_{1}} t \prod_{i_{2}=1}^{m} \frac{1}{1-\alpha_{m+i_{1}+i_{2}} t \prod_{i_{3}=1}^{m} \frac{1}{1-\cdots}}}}
$$

with coefficients $\left(\alpha_{i}\right)_{i \geq m}=(c(q+\lambda), \underbrace{b, \ldots, b}_{m}, c(q+\lambda), \underbrace{2 b, \ldots, 2 b}_{m}, c(q+\lambda), \underbrace{3 b, \ldots, 3 b}_{m}, \ldots)$. In particular, for $q=0$, we have the $m$-branched Stieltjes-type continued fraction expression

$$
\sum_{n \geq 0} T_{n, 0} t^{n}=\frac{1}{1-\alpha_{m} t \prod_{i_{1}=1}^{m} \frac{1}{1-\alpha_{m+i_{1}} t \prod_{i_{2}=1}^{m} \frac{1}{1-\alpha_{m+i_{1}+i_{2}} t \prod_{i_{3}=1}^{m} \frac{1}{1-\cdots}}}}
$$

with coefficients $\left(\alpha_{i}\right)_{i \geq m}=(c \lambda, \underbrace{b, \ldots, b}_{m}, c \lambda, \underbrace{2 b, \ldots, 2 b}_{m}, c \lambda, \underbrace{3 b, \ldots, 3 b}_{m}, \ldots)$.

(viii) The row sequence $\left(T_{n, k}\right)_{k=0}^{n}$ is a PF sequence for $\{b, c, d, \lambda\} \subseteq \mathbb{R}^{\geq 0}$.

Proof. By taking $a$ with $-a$ and $b$ with $-b$ in Proposition 4.4 , we have $\left[T_{n, k}\right]_{n, k}$ is the exponential Riordan array $\left(g(t) e^{\lambda f(t)}, f(t)\right)$, where

$$
g(t)=(1-a b t)^{-d}, \quad f(t)=c\left[\frac{(1-a b t)^{-\frac{1}{a}}-1}{b}\right]
$$

So we immediately get (i).

It is easy to get that the compositional inverse of $f(t)$ is

$$
\bar{f}(t)=\frac{1-\left(1+\frac{b t}{c}\right)^{-a}}{a b} .
$$


Clearly, both $g(t)$ for $d \in \mathbb{N}$ and

$$
1 / \bar{f}^{\prime}(t)=c\left(1+\frac{b}{c} t\right)^{a+1}
$$

are Pólya frequency functions. Thus, for $d \in \mathbb{N}$, we can immediately get $(b, \lambda)$-total positivity in (ii) by Theorem 3.6. For $c=b$, applying Theorem 1.7 to the exponential Riordan array $\left(g(t) e^{\lambda f(t)}, f(t)\right)$, we get the results in (iii)-(vi).

For (vii), by taking $q \rightarrow c(q+\lambda) / b$ and $t \rightarrow b t$, it suffices to prove for the reduced case $b=c=1$ and $\lambda=0$ that

$$
\sum_{n \geq 0} T_{n}(q) t^{n}=\frac{1}{1-\alpha_{m} t \prod_{i_{1}=1}^{m} \frac{1}{1-\alpha_{m+i_{1}} t \prod_{i_{2}=1}^{m} \frac{1}{1-\alpha_{m+i_{1}+i_{2}} t \prod_{i_{3}=1}^{m} \frac{1}{1-\cdots}}}}
$$

with coefficients

$$
\left(\alpha_{i}\right)_{i \geq m}=(q, \underbrace{1, \ldots, 1}_{m}, q, \underbrace{2, \ldots, 2}_{m}, q, \underbrace{3, \ldots, 3}_{m}, \ldots) .
$$

For the reduced case, we have

$$
\frac{1}{\bar{f}^{\prime}(t)}=(1+t)^{m}
$$

Hence we immediately get the continued fraction expansion for the reduced case by Theorem 1.6 (vi).

(viii) In order to prove that $\left(T_{n, k}\right)_{k=0}^{n}$ is a $\mathrm{PF}$ sequence, it suffices to prove that $T_{n}(q)$ has only real zeros. By the recurrence relation (4.17), we have

$$
T_{n}(q)=[a b(n-1)+a b d+c \lambda+c q] T_{n-1}(q)+b(q+\lambda) T_{n-1}^{\prime}(q)
$$

for $n \geq 1$ and $T_{0}(q)=1$. In the following, we will prove a stronger result that all zeros of $T_{n}(q)$ are real numbers and not more than $-\lambda$. It is obvious for $n=0,1$. We assume that it is true for $n-1$. Denote the zeros of $T_{n-1}(q)$ by $r_{i}$ for $1 \leq i \leq n-1$. It follows from the recurrence relation (4.18) that

$$
\operatorname{sign}\left[T_{n}\left(r_{i}\right)\right]=(-1)^{i}
$$

for $1 \leq i \leq n-1$. Note that coefficients of $T_{n}(q)$ are nonnegative and $T_{n}(-\lambda) \geq 0$. Then we get that $T_{n}(q)$ has $n$ real zeros denoted by $z_{1} \geq z_{2} \geq \ldots \geq z_{n}$ such that

$$
-\lambda \geq z_{1} \geq r_{1} \geq z_{2} \geq r_{2} \geq \ldots \geq r_{n-1} \geq z_{n}
$$

This completes the proof.

Remark 4.8. For the array in (4.17), we can give other sufficient conditions for total positivity. For example, for the exponential Riordan array $\left(g(t) e^{\lambda f(t)}, f(t)\right)$ in Proposition 4.7 , it is easy to get

$$
Z(t)=(a b d+\lambda c+\lambda b t)\left(1+\frac{b}{c} t\right)^{a}, A(t)=(c+b t)\left(1+\frac{b}{c} t\right)^{a} .
$$


By taking $\varphi(t)=\left(1+\frac{b}{c} t\right)^{a}$ in Theorem 3.9, we get its production matrix

$$
P=\left(\prod_{i=1}^{a}\left[\begin{array}{cccccc}
1 & & & & \\
\frac{b}{c} & 1 & & & \\
& \frac{2 b}{c} & 1 & & \\
& & \frac{3 b}{c} & 1 & \\
& & & \ddots & \ddots
\end{array}\right] \Lambda\left[\begin{array}{cccc}
a b d+c \lambda & c & & \\
b \lambda & a b d+c \lambda+b & 2 c & \\
& b \lambda & a b d+c \lambda+2 b & \ddots \\
& & \ddots & \ddots
\end{array}\right] \Lambda^{-1} .\right.
$$

Note that the tridiagonal matrix

$$
\left[\begin{array}{cccc}
a b d+c \lambda & c & & \\
b \lambda & a b d+c \lambda+b & 2 c & \\
& b \lambda & a b d+c \lambda+2 b & \ddots \\
& & \ddots & \ddots
\end{array}\right]
$$

is TP for $b>c$ and $\frac{a b d}{b-c} \geq \lambda>0$ (see [73, Proposition 3.1] for instance) and is $(b, d)$-TP for $\lambda=0$ and $c>0$. Hence we get the corresponding total positivity of $\left[T_{n, k}\right]_{n \geq 0}$ and $\left[T_{i+j}(q)\right]_{i, j}$ by Theorem $3.9(\mathrm{i})$.

Remark 4.9. For $\lambda=0$ and $c=1$, we have many classical combinatorial numbers as the special cases of $T_{n, k}$, e.g., the Stirling number of the second kind, the Carlitzs degenerate Stirling number [14], the Howards weighted degenerate Stirling number [27], the Todorovs number [60], and the Ahuja-Ennekings associated Lah number [36].

Let $\left[\mathcal{T}_{n, k}\right]_{n, k}$ be defined by (4.17). For the reciprocal generalized Lah polynomial $T_{n}^{*}(q)=q^{n} T_{n}(1 / q)$ and the reciprocal generalized Lah numbers $T_{n, k}^{*}=T_{n, n-k}$, by Proposition 4.7 , we immediately get the following reciprocal result.

Proposition 4.10. Let $a \in \mathbb{N}$. Then we have the following results.

(i) The reciprocal generalized Lah triangle $\left[T_{n, k}^{*}\right]_{n, k \geq 0}$ satisfies the next recurrence relation

$T_{n, k}^{*}=c T_{n-1, k}^{*}+[a b(n-1)+b(n-k)+a b d+c \lambda] T_{n-1, k-1}^{*}+b \lambda(n-k+1) T_{n-1, k-2}^{*}$

for $n, k \geq 1$, where $T_{0,0}^{*}=1$.

(ii) The exponential generating function of $T_{n}^{*}(q)$ can be written as

$$
\sum_{n \geq 0} T_{n}^{*}(q) \frac{t^{n}}{n !}=g(q t) \exp ((q \lambda+1) f(q t) / q) .
$$

(iii) For $c=b$, the sequence $\left(T_{n}^{*}(q)\right)_{n \geq 0}$ is $(b, d, \lambda, q)$-SM and $3-(b, d, \lambda, q)$-log-convex. In particular, $\left(T_{n, n}^{*}\right)_{n \geq 0}$ is $(b, d, \lambda)-S M$ and 3 - $(b, d, \lambda)$-log-convex.

(iv) Let $c=b$. The convolution $z_{n}=\sum_{k \geq 0} T_{n, k}^{*} x_{k} y_{n-k}$ preserves Stieltjes moment property of sequences for $\{b, d, \lambda\} \subseteq \mathbb{R}^{\geq 0}$; 
(v) Let $a+1=m$ and $d=0$. We have the $m$-branched Stieltjes-type continued fraction expansion

$$
\sum_{n \geq 0} T_{n}^{*}(q) t^{n}=\frac{1}{1-\alpha_{m} t \prod_{i_{1}=1}^{m} \frac{1}{1-\alpha_{m+i_{1}} t \prod_{i_{2}=1}^{m} \frac{1}{1-\alpha_{m+i_{1}+i_{2}} t \prod_{i_{3}=1}^{m} \frac{1}{1-\cdots}}}}
$$

with coefficients

$$
\left(\alpha_{i}\right)_{i \geq m}=(c(1+q \lambda), \underbrace{b q, \ldots, b q}_{m}, c(1+q \lambda), \underbrace{2 b q, \ldots, 2 b q}_{m}, c(1+q \lambda), \underbrace{3 b q, \ldots, 3 b q}_{m}, \ldots) .
$$

(iv) Let $a+1=m$ and $d=0$. We have

$$
\sum_{n \geq 0} T_{n, n}^{*} t^{n}=\frac{1}{1-\alpha_{m} t \prod_{i_{1}=1}^{m} \frac{1}{1-\alpha_{m+i_{1}} t \prod_{i_{2}=1}^{m} \frac{1}{1-\alpha_{m+i_{1}+i_{2}} t \prod_{i_{3}=1}^{m} \frac{1}{1-\cdots}}}}
$$

with coefficients $\left(\alpha_{i}\right)_{i \geq m}=(c \lambda, \underbrace{b, \ldots, b}_{m}, c \lambda, \underbrace{2 b, \ldots, 2 b}_{m}, c \lambda, \underbrace{3 b, \ldots, 3 b}_{m}, \ldots)$.

(v) The row sequence $\left(T_{n, k}^{*}\right)_{k=0}^{n}$ is a PF sequence for $\{b, c, d, \lambda\} \subseteq \mathbb{R}^{\geq 0}$.

Similar to Proposition 4.6, we also have the next result.

Proposition 4.11. Let $a \in \mathbb{N}$ and $c \in \mathbb{R}^{+}$. Assume that a triangular array $\left[\widetilde{T}_{n, k}\right]_{n, k \geq 0}$ satisfies the following recurrence relation

$$
\widetilde{T}_{n, k}=c k \widetilde{T}_{n-1, k-1}+[a b(n-1)+b k+a b d+c \lambda] \widetilde{T}_{n-1, k}+b \lambda \widetilde{T}_{n-1, k+1}
$$

for $n, k \geq 1$, where $\widetilde{T}_{0,0}=1$. Let $\widetilde{T}_{n}(q)=\sum_{k \geq 0} \widetilde{T}_{n, k} q^{k}$. Then we have the following results.

(i) An explicit formula of $\widetilde{T}_{n, k}$ can be written as

$$
\widetilde{T}_{n, k}=a^{n} \sum_{j \geq 0} \sum_{i=0}^{k+j} \frac{(-1)^{n+k+j-i} b^{n-k-j} c^{k+j} \lambda^{j}}{j !}\left(\begin{array}{c}
k+j \\
i
\end{array}\right)\left(-\frac{i+a d}{a}\right)_{n}
$$

for $n, k \geq 1$.

(ii) For $c=b$, the lower-triangular matrix $\left[\widetilde{T}_{n, k}\right]_{n, k \geq 0}$ is $(b, d, \lambda)-T P$.

(iii) For $d=0$, the exponential generating function is

$$
1+\sum_{n \geq 1} \widetilde{T}_{n}(q) \frac{t^{n}}{n !}=\frac{1}{1+\frac{c}{b}\left[1-(1-a b t)^{-\frac{1}{a}}\right](q+\lambda)} .
$$

(iv) Let $c=b$. The convolution $z_{n}=\sum_{k \geq 0} \widetilde{T}_{n, k} x_{k} y_{n-k}$ preserves Stieltjes moment property of sequences for $\{b, d, \lambda\} \subseteq \mathbb{R}^{\geq 0}$. 


\subsection{Rook polynomials and signless Laguerre polynomials}

Let $\mathfrak{S}_{n}(q)$ denote the rook polynomial of a square of side $n$. It is well-known that it is given by

$$
\mathfrak{S}_{n}(q)=\sum_{k=0}^{n}\left(\begin{array}{l}
n \\
k
\end{array}\right)^{2} k ! q^{k},
$$

see [46, Chapter 3. Problems 18] for instance, and it can also be viewed as the matching polynomial of the complete bipartite graph $K_{n, n}$. It has only real zeros in terms of the rook theory or matching polynomials. The rook polynomials $\mathfrak{S}_{n}(q)$ form a strongly $q$-logconvex sequence [74]. Recently, Wang and Zhu [63] proved that the transformation

$$
z_{n}=\sum_{k=0}^{n}\left(\begin{array}{l}
n \\
k
\end{array}\right)^{2} x_{k}
$$

preserves the Stieltjes moment property for real numbers. Note for fixed $q>0$ that $\left(k ! q^{k}\right)_{k \geq 0}$ is a Stieltjes moment sequence. Thus we get for $n \geq 0$ that

$$
\mathfrak{S}_{n}(q)=\sum_{k=0}^{n}\left(\begin{array}{l}
n \\
k
\end{array}\right)^{2} k ! q^{k}
$$

form a Stieltjes moment sequence for fixed $q>0$. These support the following stronger conjecture of Sokal.

Conjecture 4.12. [56] The sequence $\left(\mathfrak{S}_{n}(q)\right)_{n \geq 0}$ is q-Stieltjes moment, that is to say that the Hankel matrix $\left[\mathfrak{S}_{i+j}(q)\right]_{i, j}$ is $q-T P$.

As an application of our results, we will demonstrate Conjecture 4.12.

Proposition 4.13. The sequence $\left(\mathfrak{S}_{n}(q)\right)_{n \geq 0}$ is q-Stieltjes moment.

The rook polynomials $\mathfrak{S}_{n}(q)$ are closely related to the famous Laguerre polynomials. For the Laguerre polynomial $L_{n}^{(\alpha)}(q)$ with $\alpha \geq-1$ (see [2] for instance), its exponential generating function is

$$
\sum_{n \geq 0} L_{n}^{(\alpha)}(q) \frac{t^{n}}{n !}=\frac{1}{(1-t)^{\alpha+1}} \exp \left(\frac{q t}{t-1}\right) .
$$

In addition, it can be given by

$$
L_{n}^{(\alpha)}(q)=\sum_{k=0}^{n}\left(\begin{array}{l}
n+\alpha \\
n-k
\end{array}\right) \frac{n !}{k !}(-q)^{k}
$$

for $n \geq 0$. Let $\widetilde{L}_{n}^{(\alpha)}(q)=L_{n}^{(\alpha)}(-q)$ for $n \geq 0$. It is obvious for $\alpha=0$ that

$$
\mathfrak{S}_{n}(q)=q^{n} \widetilde{L}_{n}^{(0)}(1 / q)
$$

In addition, for $\alpha=-1$, the Lah polynomial $\mathcal{L}_{n}(q)=\widetilde{L}_{n}^{(-1)}(q)$. For signless Laguerre polynomials $\widetilde{L}_{n}^{(\alpha)}(q)$, we have the following result, which also implies Proposition 4.13. 
Proposition 4.14. Let $\alpha \geq-1$ and $\widetilde{L}_{n}^{(\alpha)^{*}}(q)=q^{n} \widetilde{L}_{n}^{(\alpha)}(1 / q)$. Then we have

(i) both $\left(\widetilde{L}_{n}^{(\alpha)}(q)\right)_{n \geq 0}$ and $\left(\widetilde{L}_{n}^{(\alpha)^{*}}(q)\right)_{n \geq 0}$ are $q$-Stieltjes moment and 3 - $q$-log-convex;

(ii) the triangular matrix $\left[\left(\begin{array}{c}n+\alpha \\ n-k\end{array}\right) \frac{n !}{k !}\right]_{n, k}$ is TP;

(iii) the convolution

$$
z_{n}=\sum_{k \geq 0}\left(\begin{array}{c}
n+\alpha \\
n-k
\end{array}\right) \frac{n !}{k !} x_{k} y_{n-k}
$$

preserves the SM property.

Proof. By (4.19), we have

$$
\sum_{n \geq 0} \widetilde{L}_{n}^{(\alpha)}(q) \frac{t^{n}}{n !}=\frac{1}{(1-t)^{\alpha+1}} \exp \left(\frac{q t}{1-t}\right) .
$$

Let us consider the exponential Riordan array $(g(t), f(t))$, where

$$
g(t)=\frac{1}{(1-t)^{\alpha+1}} \exp \left(\frac{q t}{1-t}\right)=(1+f(t))^{\alpha+1} \exp (q f(t)), \quad f(t)=\frac{t}{1-t} .
$$

Hence we get that

$$
\frac{1}{(1+t) \bar{f}^{\prime}(t)}=1+t
$$

is a PF function. So by Proposition 4.7 or Theorem 1.7, the desired results are immediate.

\subsection{Idempotent numbers}

It is well-known that idempotent numbers

$$
I_{n, k}=\left(\begin{array}{l}
n \\
k
\end{array}\right) k^{n-k}
$$

satisfy

$$
\exp (q t \exp (t))=\sum_{n \geq 0} \sum_{k=0}^{n} I_{n, k} q^{k} \frac{t^{n}}{n !} .
$$

The inverse of the idempotent triangle $\left[\left(\begin{array}{l}n \\ k\end{array}\right) k^{n-k}\right]_{n, k \geq 0}$ is $\left[(-1)^{n-k}\left(\begin{array}{c}n-1 \\ k-1\end{array}\right) n^{n-k}\right]_{n, k \geq 1}$, where $\left(\begin{array}{c}n-1 \\ k-1\end{array}\right) n^{n-k}$ counts the number of rooted labeled trees on $n+1$ vertices with a root degree $k$, see [55, A137452]. It is known that the compositional inverse of $t \exp (t)$ is the Lambert function $W(t)=\sum_{n \geq 1} \frac{(-n)^{n-1} t^{n}}{n !}$ (This can be obtained in terms of the Lagrange inversion formula, see [20, p. 152] for instance). Thus

$$
\exp (-q W(-t))=1+\sum_{n \geq 1} \sum_{k=1}^{n}\left(\begin{array}{l}
n-1 \\
k-1
\end{array}\right) n^{n-k} q^{k} \frac{t^{n}}{n !}
$$

and the compositional inverse of $-W(-t)$ is $t \exp (-t)$. Obviously, $\frac{1}{(t \exp (-t))^{\prime}}=\frac{\exp (t)}{1-t}$ is a $\mathrm{PF}$ function. Then the following is immediate from Theorem 1.6. 
Proposition 4.15. (i) Matrices $\left[\left(\begin{array}{l}n \\ k\end{array}\right) k^{n-k}\right]_{n, k \geq 0}$ and $\left[\left(\begin{array}{c}n-1 \\ k-1\end{array}\right) n^{n-k}\right]_{n, k \geq 1}$ are TP.

(ii) Matrices $\left[\left(\begin{array}{l}n \\ k\end{array}\right) k^{n-k} k !\right]_{n, k \geq 0}$ and $\left[\left(\begin{array}{c}n-1 \\ k-1\end{array}\right) n^{n-k} k !\right]_{n, k \geq 1}$ are TP.

(iii) The sequence $\left(\mathcal{I}_{n}(q)\right)_{n \geq 0}$ is $q$-SM and 3-q-log-convex, where $\mathcal{I}_{n}(q)=\sum_{k=1}^{n}\left(\begin{array}{c}n-1 \\ k-1\end{array}\right) n^{n-k} q^{k}$.

(iv) The sequence $\left((1+q n)^{n-1}\right)_{n \geq 1}$ are $q$-SM and $3-q$-log-convex.

(v) The sequence $\left(n^{n-1}\right)_{n \geq 1}$ is $S M$.

(vi) Both convolutions $z_{n}=\sum_{k \geq 1}\left(\begin{array}{c}n-1 \\ k-1\end{array}\right) n^{n-k} x_{k} y_{n-k}$ and $z_{n}=\sum_{k \geq 0}\left(\begin{array}{c}n \\ k\end{array}\right)(n+1)^{n-k} x_{k} y_{n-k}$ preserve Stieltjes moment property of sequences.

\subsection{Some numbers related to binomial coefficients}

It is well-known that the Pascal triangle $\left[\left(\begin{array}{l}n \\ k\end{array}\right)\right]_{n, k \geq 0}$ is TP, see [30] for instance. Let $C_{k}=$ $\left(\begin{array}{c}n+c k \\ m+d k\end{array}\right)$, where $n \geq m$. Then for integers $d>c>0$, the Toeplitz matrix $\left[C_{i-j}\right]_{i, j \geq 0}$ of the finite sequence $\left(C_{k}\right)_{k}$ is TP, which was conjectured in [59] and proved in [65]. Recently, we also proved for real numbers $c \geq d>0$ that the Hankel matrix $\left[C_{i+j}\right]_{i, j \geq 0}$ of the infinite sequence $\left(C_{k}\right)_{k}$ is TP [71]. In the following, we will present more results for total positivity related to binomial coefficients.

Proposition 4.16. Let $c$ and $d$ be integers and $\{m, n\} \subseteq \mathbb{N}$. Then

(i) for $d>0$ and $d \geq c$, the low-triangular matrices $\left[\left(\begin{array}{l}n \\ k\end{array}\right)\left(\begin{array}{c}n+c k \\ m+d k\end{array}\right)(n-k) !\right]_{n, k \geq 0}$ and $\left[\left(\begin{array}{c}n+c k \\ m+d k\end{array}\right)\right]_{n, k \geq 0}$ are TP;

(ii) for $d \leq-1$ and $c \in \mathbb{N}$, the low-triangular matrices $\left[\left(\begin{array}{c}m \\ k\end{array}\right)\left(\begin{array}{c}n+c k \\ m+d k\end{array}\right)(m-k) !\right]_{m, k \geq 0}$ and $\left[\left(\begin{array}{c}n+c k \\ m+d k\end{array}\right)\right]_{m, k \geq 0}$ are TP.

Proof. By Lemma 3.5, it suffices to prove that matrices $\left[\left(\begin{array}{l}n \\ k\end{array}\right)\left(\begin{array}{c}n+c k \\ m+d k\end{array}\right)(n-k) !\right]_{n, k \geq 0}$ in (i) and $\left[\left(\begin{array}{c}m \\ k\end{array}\right)\left(\begin{array}{c}n+c k \\ m+d k\end{array}\right)(m-k) !\right]_{m, k \geq 0}$ in (ii) are TP. Note that

$$
\begin{aligned}
\left(\begin{array}{l}
n \\
k
\end{array}\right)\left(\begin{array}{c}
n+c k \\
m+d k
\end{array}\right)(n-k) ! & =\frac{n !}{k !}\left(\begin{array}{c}
n+c k \\
(n-m)+(c-d) k
\end{array}\right) \\
& =\frac{n !}{k !}\left(\begin{array}{c}
-m-d k-1 \\
(n-m)+(c-d) k
\end{array}\right)(-1)^{(n-m)+(c-d) k} \\
& =\frac{n !}{k !}\left[t^{(n-m)+(c-d) k}\right] \frac{1}{(1-t)^{m+1+d k}} \\
& =\frac{n !}{k !}\left[t^{n}\right] \frac{t^{m}}{(1-t)^{m+1}}\left(\frac{t^{d-c}}{(1-t)^{d}}\right)^{k} \\
& =\left(\frac{t^{m}}{(1-t)^{m+1}}, \frac{t^{d-c}}{(1-t)^{d}}\right)
\end{aligned}
$$




$$
\begin{aligned}
\left(\begin{array}{c}
m \\
k
\end{array}\right)\left(\begin{array}{c}
n+c k \\
m+d k
\end{array}\right)(m-k) ! & =\frac{m !}{k !}\left(\begin{array}{c}
n+c k \\
m+d k
\end{array}\right) \\
& =\frac{m !}{k !}\left[t^{m+d k}\right](1+t)^{n+c k} \\
& =\frac{m !}{k !}\left[t^{m}\right](1+t)^{n}\left(t^{-d}(1+t)^{c}\right)^{k} \\
& =\left((1+t)^{n}, t^{-d}(1+t)^{c}\right)
\end{aligned}
$$

where $\frac{t^{m}}{(1-t)^{m+1}}, \frac{t^{d-c}}{(1-t)^{d}},(1+t)^{n}$ and $t^{-d}(1+t)^{c}$ are Pólya frequency functions under our conditions. Thus, it follows from Theorem 3.6 and Remark 3.7 that $\left[\left(\begin{array}{l}n \\ k\end{array}\right)\left(\begin{array}{c}n+c k \\ m+d k\end{array}\right)(n-k) !\right]_{n, k \geq 0}$ in (i) and $\left[\left(\begin{array}{c}m \\ k\end{array}\right)\left(\begin{array}{c}n+c k \\ m+d k\end{array}\right)(m-k) !\right]_{m, k \geq 0}$ in (ii) are TP. The proof is complete.

\section{Acknowledgements}

The author would like to thank the anonymous reviewer for many valuable remarks and suggestions to improve the original manuscript.

\section{References}

[1] Wm.R. Allaway, Extensions of Sheffer polynomial sets, SIAM J. Math. Anal. 10 (1979) 38-48.

[2] G.E. Andrews, R. Askey and R. Roy, Special Functions, Cambridge University Press, Cambridge, 1999.

[3] W.A. Al-Salam, A. Verma, Generalized Sheffer polynomials, Duke Math. J. 37 (1970) $361-365$.

[4] P. Barry, Riordan arrays, Orthogonal polynomials as moments, and Hankel transforms, J. Integer Seq. 14 (2011), Article 11.2.2.

[5] E.A. Bender and E.R. Canfield, Log-concavity and related properties of the cycle index polynomials, J. Combin. Theory Ser. A 74 (1996) 57-70.

[6] P. Brändén, On linear transformations preserving the Pólya frequency property, Trans. Amer. Math. Soc. 358 (2006) 3697-3716.

[7] P. Brändén, Unimodality, log-concavity, real-rootedness and beyond, in Handbook of Combinatorics (M. Bona, ed.), CRC Press, 2015, pp. 437-483.

[8] F. Brenti, Unimodal, log-concave, and Pólya frequency sequences in combinatorics, Mem. Amer. Math. Soc. 413 (1989).

[9] F. Brenti, Log-concave and unimodal sequences in algebra, combinatorics, and geometry: an update, Contemp. Math. 178 (1994) 71-89.

[10] F. Brenti, Combinatorics and total positivity, J. Combin. Theory Ser. A 71 (1995) $175-218$. 
[11] F. Brenti, The applications of total positivity to combinatorics, and conversely, in Total Positivity and its Applications, (M. Gasca, C. A. Micchelli, eds.), Kluwer Academic Pub., Dordrecht, The Netherlands, 1996, 451-473.

[12] L.M. Butler, The $q$-log concavity of q-binomial coeffcients, J. Combin. Theory Ser. A 54 (1990) 54-63.

[13] D. Callan, A combinatorial survey of identities for the double factorial, arxiv:0906.1317v1.

[14] L. Carlitz, Degenerate Stirling, Bernoulli and Eulerian numbers, Utilitas Math. 15 (1979) 51-88.

[15] L. Carlitz, A note on the Bessel polynomials, Duke. Math. J. 23 (1957) 151-162.

[16] W.Y.C. Chen, L.X.W. Wang and A.L.B. Yang, Recurrence relations for strongly q-log-convex polynomials, Canad. Math. Bull. 54 (2011) 217-229.

[17] X. Chen, H. Liang, Y. Wang, Total positivity of recursive matrices, Linear Algebra Appl. 471 (2015) 383-393.

[18] X. Chen, H. Liang, Y. Wang, Total positivity of Riordan arrays, European J. Combin. 46 (2015) 68-74.

[19] X. Chen, Y. Wang, Notes on the total positivity of Riordan arrays, Linear Algebra Appl. 569 (2019) 156-161.

[20] L. Comtet, Advanced combinatorics, D. Reidel Publishing Co., Dordrecht, 1974.

[21] E. Deutsch, L. Ferrari, S. Rinaldi, Production matrices and Riodan arrays, Ann. Comb. 13 (2009) 65-85.

[22] E. Deutsh, L. Shapiro, Exponential Riordan arrays, Lecture Notes, Nankai University, 2004, available electronically at http://www.combinatorics.net/ppt2004/Louis.

[23] I. Gessel and G. Viennot, Binomial determinants, path, and hook length formulae, Adv. Math. 58 (1985) 300-321.

[24] E. Grosswald, On some algebraic properties of the Bessel polynomials, Trans. Amer. Math. Soc. 71 (1951) 197-210.

[25] B.G. Hansen, On log-concave and log-convex infinitely divisible sequences and densities, Ann. Probab. 16 (1988) 1832-1839.

[26] B.G. Hansen, F.W Steutel, On moment sequences and infinitely divisible sequences, J. Math. Anal. Appl. 136 (1988) 304-313.

[27] F. T. Howard, Degenerate weighted Stirling numbers, Discrete Math. 57 (1985) 4558.

[28] L.L. Liu, Y. Wang, On the log-convexity of combinatorial sequences, Adv. in Appl. Math. 39 (2007) 453-476. 
[29] O.M. Katkova, A.M. Vishnyakova, On sufficient conditions for the total positivity and for the multiple positivity of matrices, Linear Algebra Appl. 416 (2006) 1083-1097.

[30] S. Karlin, Total Positivity, Vol. I, Stanford University Press, Stanford, 1968.

[31] C. Krattenthaler, On the $q$-log-concavity of Gaussian binomial coefficients, Monatsh. Math. 107 (1989) 333-339.

[32] H.L. Krall, O. Frink, A new class of orthogonal polynomials, Trans. Amer. Math. Soc. 65 (1945) 100-115.

[33] P. Leroux, Reduced matrices and q-log-concavity properties of q-Stirling numbers, J. Combin. Theory Ser. A 54 (1990) 64-84.

[34] T. Mikosch, Non-Life Insurance Mathematics. An Introduction with the Poisson Process. 2nd ed. Springer, Heidelberg, 2009.

[35] P. Mongelli, Total positivity properties of Jacobi-Stirling numbers, Adv. in. Appl. Math. 48 (2012) 354-364.

[36] S.B. Nandi and S. K. Dutta, On associated and generalized Lah numbers and applications to discrete distribution, Fibonacci Quart. (1987) 128-136.

[37] L. Mu, S.-N. Zheng, On the total positivity of Delannoy-like triangles, J. Integer Seq. 20 (2017) 17.1.6.

[38] Q. Pan, J. Zeng, On total positivity of Catalan-Stieltjes matrices, The Electronic J. Combin. 23 (4) (2016) P.4.33.

[39] M. Pétréolle, A.D. Sokal, Lattice paths and branched continued fractions. II. Multivariate Lah polynomials and Lah symmetric functions, preprint (2019), arXiv:1907.02645v1.

[40] M. Pétréolle, A.D. Sokal and B.-X. Zhu, Lattice paths and branched continued fractions: An infinite sequence of generalizations of the Stieltjes-Rogers and ThronRogers polynomials, with coefficientwise Hankel-total positivity, to appear in Mem. Amer. Math. Soc., arXiv:1807.03271.

[41] A. Pinkus. Totally Positive Matrices, Cambridge University Press, Cambridge, 2010.

[42] G. Pólya, Kombinatorisch Anzahlbestimmungen für Gruppen, Graphen und chemische Verbindungen, Acta Math. 68 (1937) 145-253.

[43] G. Pólya and G. Szegö, Problems and Theorems in Analysis, Vol. II, Springer-Verlag, Berlin, 1976.

[44] J.H. Redfield, The theory of group-reduced distributions, Amer. J. Math. 49 (1927) 433-455.

[45] K. Rietsch, Totally positive Toeplitz matrices and quantum cohomology of partial flag varieties, J. Amer. Math. Soc. 16(2) (2003) 363-392. 
[46] J. Riordan, Combinatorial Identities, New York, 1979.

[47] S. Roman, G.-C. Rota, The umbral calculus, Adv. Math. 27 (2) (1978) 95-188.

[48] G.-C. Rota, Finite Operator Calculus, Academic Press, New York, 1975.

[49] G.-C. Rota, D. Kahaner, A. Odlyzko, On the foundations of combinatorial theory. VIII. Finite operator calculus, J. Math. Anal. Appl. 42 (1973) 684-760.

[50] B.E. Sagan, Log concave sequences of symmetric functions and analogs of the JacobiTrudi determinants, Trans. Amer. Math. Soc. 329 (1992) 795-811.

[51] K.-I. Sato, Lévy Processes and Infinitely Divisible Distributions, Cambridge University Press, Cambridge, UK, 1999.

[52] E. Schirmacher, Log-concavity and the exponential formula, J. Combin. Theory Ser. A 85 (1999) 127-134.

[53] I.M. Sheffer, Some properties of polynomial sets of type zero, Duke Math. J. 5 (1939) $590-622$.

[54] J.A. Shohat and J.D. Tamarkin, The Problem of Moments, Amer. Math. Soc., New York, 1943.

[55] N.J.A. Sloane, The On-Line Encyclopedia of Integer Sequences, http://oeis.org.

[56] A.D. Sokal, Continued fractions and Hankel-total positivity, 15th International Symposium on Orthogonal Polynomials, Special Functions and Applications, Hagenberg, Austria, 23 July 2019, https://www3.risc.jku.at/conferences/opsfa2019/talk/sokal/ pdf.

[57] J. Stembridge, Nonintersecting paths, pfaffians, and plane partitions, Adv. Math. 83 (1990) 96-131.

[58] J.F. Steffensen, The poweroid, an extension of the mathematical notion of power, Acta Math. 73 (1941) 333-366.

[59] X.-T. Su, Y. Wang, On the unimodality problems in Pascal triangle, Electron. J. Combin. 15 (2008), \#R113.

[60] P. G. Todorov, Taylor expansions of analytic functions related to $(1+z)^{x}-1$, J. Math. Anal. Appl. 132 (1988) 264-280.

[61] Y. Wang, A.L.B. Yang, Total positivity of Narayana matrices, Discrete Math. 341 (2018) 1264-1269.

[62] Y. Wang, Y.-N. Yeh, Polynomials with real zeros and Pólya frequency sequences, J. Combin. Theory Ser. A 109 (2005) 63-74.

[63] Y. Wang, B.-X. Zhu, Log-convex and Stieltjes moment sequences, Adv. in Appl. Math. 81 (2016) 115-127. 
[64] D.V. Widder, The Laplace Transform, Princeton University Press, Princeton, 1946.

[65] Y. Yu, Confirming two conjectures of Su and Wang on binomial coefficients, Adv. in. Appl. Math. 43(4) (2009) 317-322.

[66] B.-X. Zhu, Log-convexity and strong $q$-log-convexity for some triangular arrays, Adv. in Appl. Math. 50 (2013) 595-606.

[67] B.-X. Zhu, Some positivities in certain triangular array, Proc. Amer. Math. Soc. 142(9) (2014) 2943-2952.

[68] B.-X. Zhu, q-log-convexity from linear transformations and polynomials with real zeros, European J. Combin. 73 (2018) 231-246.

[69] B.-X. Zhu, Positivity of iterated sequences of polynomials, SIAM J. Discrete Math. 32 (2018) 1993-2010.

[70] B-X. Zhu, Positivity and continued fractions from the binomial transformation, Proc. Roy. Soc. Edinburgh Sect. A 149 (2019) 831-847.

[71] B-X. Zhu, Hankel-total positivity of some sequences, Proc. Amer. Math. Soc. 147 (2019) 4673-4686.

[72] B.-X. Zhu, A generalized Eulerian triangle from staircase tableaux and tree-like tableaux, J. Combin. Theory Ser. A 172 (2020), Article 105206, 32 pp.

[73] B.-X. Zhu, Stieltjes moment properties and continued fractions from combinatorial triangles, preprint, Adv. in. Appl. Math. 130 (2021), Article 102232, 33pp. arXiv:2007.14924.

[74] B.-X. Zhu, H. Sun, Linear transformations preserving the strong $q$-log-convexity of polynomials, Electron. J. Combin. 22 (3) (2015), Paper P3.26. 